\title{
Oscillatory Entrainment of Primary Somatosensory Cortex Encodes Visual Control of Tactile Processing
}

\author{
Kay Sieben, ${ }^{1}$ Brigitte Röder, ${ }^{2}$ and Ileana L. Hanganu-Opatz ${ }^{1}$ \\ ${ }^{1}$ Developmental Neurophysiology, Center for Molecular Neurobiology, University Medical Center Hamburg-Eppendorf, 20251 Hamburg, Germany, and \\ 2Biological Psychology and Neuropsychology, University Hamburg, 20146 Hamburg, Germany
}

Optimal behavior relies on the successful integration of complementary information from multiple senses. The neural mechanisms underlying multisensory interactions are still poorly understood. Here, we demonstrate the critical role of neural network oscillations and direct connectivity between primary sensory cortices in visual-somatosensory interactions. Extracellular recordings from all layers of the barrel field in Brown Norway rats in vivo showed that bimodal stimulation (simultaneous light flash and whisker deflection) augmented the somatosensory-evoked response and changed the power of induced network oscillations by resetting their phase. Anatomical tracing revealed sparse direct connectivity between primary visual (V1) and somatosensory (S1) cortices. Pharmacological silencing of V1 diminished but did not abolish cross-modal effects on S1 oscillatory activity, while leaving the early enhancement of the evoked response unaffected. Thus, visual stimuli seem to impact tactile processing by modulating network oscillations in S1 via corticocortical projections and subcortical feedforward interactions.

\section{Introduction}

The interplay of different senses is a very efficient strategy for amplifying behaviorally relevant stimuli (Stein, 2012). Integration of visual and tactile stimuli into a coherent percept is mandatory for day-to-day life, especially during visually guided actions. This is impressively demonstrated by the perceptual illusion of "rubber arm": a dummy arm aligned with one's own body and tactually stimulated together with the own hand is perceived as belonging to the one's own body (Botvinick and Cohen, 1998).

Originally, it was assumed that the integration of inputs across senses follows hierarchically organized pathways and mainly involves higher cortical areas and some subcortical nuclei (Meredith and Stein, 1983; Rodgers et al., 2008; Stein and Stanford, 2008; Deeg and Aizenman, 2011). Neurons in multisensory brain regions receive convergent inputs from multiple senses and encode perceptual information by enhancing or depressing their firing in response to cross-modal versus unimodal stimuli (Meredith, 2002; Stein and Rowland, 2011). However, the classical convergence view cannot account for all features of multisensory processing (Driver and Spence, 2000). Experimental

Received Sept. 18, 2012; revised Jan. 30, 2013; accepted Feb. 19, 2013.

Author contributions: B.R. and I.L.H.-0. designed research; K.S. and I.L.H.-0. performed research; K.S. analyzed data; K.S., B.R., and I.L.H.-O. wrote the paper.

This work was supported by the excellence initiative of city Hamburg ("neurodapt!" to K.S., B.R. and I.L.H.-0.), Emmy Noether-Program of German Research Foundation Grant Ha4466/3-1 to I.L.H.-0., Grant SFB 936 B05 to I.L.H.-0., and German Federal Ministry of Education and Research Grant 01GQ0809 to I.L.H.-0. We thank Drs. Shigeru Kitazawa, Andreas Engel, and Amy Wolff for valuable discussions and helpful comments on the manuscript; Achim Dahlmann for technical assistance; Fritz Kutschera and Torsten Renz for building the custom-designed stimulator; and Martin Westerberg for help with statistics.

The authors declare no competing financial interests.

Correspondence should be addressed to either Dr. lleana L. Hanganu-0patz or Dr. Kay Sieben, Developmental Neurophysiology, Center for Molecular Neurobiology, University Medical Center Hamburg-Eppendorf, Falkrenried 94, 20251 Hamburg, Germany, E-mail: hangop@zmnh.uni-hamburg.de or kay.sieben@zmnh.uni-hamburg.de.

DOI:10.1523/JNEUROSCI.4432-12.2013

Copyright $\odot 2013$ the authors $\quad 0270-6474 / 13 / 335736-15 \$ 15.00 / 0$ evidence has documented cross-modal activation in the primary sensory cortices, which traditionally have been considered as sensory-specific (Ghazanfar and Schroeder, 2006; Guzman-Martinez et al., 2012). Several attempts have been undertaken to elucidate by which means cross-modal inputs reach the primary cortices. Feedback projections from higher convergence areas have been proposed as main substrate of cross-modal activation in putative unisensory cortices (Clavagnier et al., 2004). Moreover, direct but rather sparse connections between primary sensory cortices have been identified (Beer et al., 2011), yet their contribution to multisensory processing remains largely unknown (Cappe and Barone, 2005).

In the primary sensory cortices, cross-modal interactions take place at the level of individual neurons as well as at the neuronal network level (Miller and D'Esposito, 2005; Kayser et al., 2007; Iurilli et al., 2012). For example, visual and olfactory stimuli modulate neuronal firing in the auditory cortex (Kayser et al., 2008; Cohen et al., 2011). Correlated neuronal activity within cortical networks might support the multisensory integration of firing rates. Cross-modal stimulation shapes the power and phase of oscillatory activity, enabling flexible modulation of the response's strength (Lakatos et al., 2007; Arnal et al., 2011). Synchronization in $\gamma$ frequency band, which allows rapid and transient enhancement of the mutual influence of neural populations (Wang, 2010), may provide another efficient mechanism of multisensory integration (Senkowski et al., 2008).

Although these findings highlight diverse mechanisms of cross-modal interplay, the link between anatomical substrates and complex dynamic interactions of neuronal populations during multisensory processing is still missing (Cappe and Barone, 2005). The present study aims at filling this gap by focusing on the poorly investigated visual-somatosensory interactions. For this, multisite extracellular recordings and pharmacological manipu- 
lation of S1 and V1 in vivo during unimodal or cross-modal stimulations were combined with tracing of axonal projections between both cortices. We provide evidence that corticocortical connectivity accounts for the visual modulation of oscillatory power and phase in somatosensory networks and also in combination with subcortical feedforward interactions, for supraadditive effects on evoked potentials.

\section{Materials and Methods}

\section{Surgical preparation}

All experiments were performed in compliance with the German laws and the guidelines of the European Community for the use of animals in research and were approved by the local ethical committee. Brown Norway rats were obtained from Charles River and housed individually in the animal facility of University Medical Center with a $12 \mathrm{~h}$ light/12 h dark cycle and fed ad libitum. Male and female rats weighing 32-41 g were used. The surgery was performed under ketamine/xylazine anesthesia (72/9.6 mg/kg body weight, i.p.; Ketavet, Pfizer electromagnetic valves; Rompun, Bayer). The scalp was removed, and two metal anchor bars were fixed on the nasal and occipital parts of the skull via dental cement, serving for fixation in the stereotaxic device. The bone over S1 and V1 was removed by drilling holes of $<0.5 \mathrm{~mm}$ in diameter without causing leakage of CSF or blood. According to our unpublished observations (I.L.H.-O.), such leakage damps cortical activity and neuronal firing.

\section{Recording protocols}

Extracellular recordings were performed under light urethane anesthesia ( $0.5 \mathrm{~g} / \mathrm{kg}$ body weight, i.p., Sigma-Aldrich). Body temperature, breathing rate, and pain reflexes were monitored. During recording (120-220 min after initial injection), additional urethane $(0.25-0.5 \mathrm{~g} / \mathrm{kg}$ body weight) was administered via an intramuscular catheter $(n=6$ rats). One-shank 16-channel electrodes with an interrecording site spacing of $100 \mu \mathrm{m}$ (0.5-3 M $\Omega$, Silicon Michigan probes, NeuroNexus Technologies) were perpendicularly inserted into S1 $(2.4-2.6 \mathrm{~mm}$ posterior to bregma and $5.5-5.8 \mathrm{~mm}$ from the midline) and V1 (6.9-7.1 $\mathrm{mm}$ posterior to bregma and $3.4-3.7 \mathrm{~mm}$ from the midline) of both hemispheres to a depth of 1.6 $\mathrm{mm}$. The electrodes were labeled with DiI $\left(1,1^{\prime}\right.$-dioctadecyl-3,3,3', $3^{\prime}$ tetramethyl indocarbocyanine, Invitrogen) to enable postmortem in histological sections the reconstruction of electrode tracks in S1 and V1 (see Fig. 1C). Two silver wires were inserted into the cerebellum and served as ground and reference electrodes. Local field potentials were recorded at a sampling rate of $32 \mathrm{kHz}$ using a multichannel extracellular amplifier (Digital Lynx 10S, Neuralynx) and the acquisition software Cheetah. During recording, the signal was bandpass filtered between $0.1 \mathrm{~Hz}$ and $5 \mathrm{kHz}$.

\section{Sensory stimulation}

Unimodal (light flash, whisker deflection) or bimodal stimulation was achieved using a custom-made stimulation device. During bimodal stimulation, whisker deflection and light flashes were presented simultaneously either in the same (congruent) hemifields or in opposite (incongruent) hemifields with respect to the tactile stimulus. Whiskers were stimulated with a precise timing $(0.013 \pm 0.81 \mathrm{~ms})$ by deflection through a compressed-air controlled roundline cylinder (RT/57110/M/ 10 , Norgren) gated via solenoid valves (VCA, SMC Pneumatik). To guarantee nearly noiseless and nonelectrical stimulation, the solenoid valves were placed outside the setup and isolated with foamed material. Because the strength of deflection was constant, the pattern and duration of "follow-up" whisker vibrations were also constant across trials and did not influence the cross-modal effects; 50 ms LED light flashes (300 Lx) were used for visual stimulation. A custom-made controlling device (V.115.2.09) triggered the stimuli in 4 different conditions. Unimodal visual and tactile, congruent, and incongruent simultaneous crossmodal stimulations were randomized through the controlling device and presented at interstimulus intervals of $6.5 \pm 0.5 \mathrm{~s}$. The nonstimulated eye was covered with an aluminum foil patch, and ears were additionally sealed with cotton. Each type of stimulus was presented $100 \pm 10$ times, except for stimulation under lidocaine. The reversibility of drug-induced blockade (Frostig et al., 2008) reduced the effective time for stimulation and consequently the number of stimuli to $50 \pm 10$.

\section{Blockade of V1 activity with lidocaine}

Blockade of action potentials in V1 was performed in rats mounted in the stereotaxic apparatus. A total volume of $100-300 \mathrm{nl}$ lidocaine (4\% in artificial CSF, Sigma-Aldrich) was intracortically applied at a rate of 200 $\mathrm{nl} /$ min via a $26 \mathrm{G}$ needle ( $10 \mu \mathrm{l}$ microsyringe) attached to a microsyringe pump controller (Micro4, WPI). To confine the lidocaine-induced blockade to V1, we calculated the appropriate drug volume according to the spherical volume equation (Tehovnik and Sommer, 1997) as follows:

$$
V=\frac{4}{3} \pi(r)^{3}
$$

where $V$ is the lidocaine volume and $r$ is the radius of tissue in which neurons are inactivated $\geq 90 \%$ of the time. The site of drug application was in the direct vicinity $(<0.5 \mathrm{~mm})$ of the Michigan electrode inserted into V1. In our previous studies, the application procedure was optimized to avoid side effects attributable to mechanical damage of tissue. Insertion of microsyringe alone or paired with infusion of solvent had no effect on network activity (Janiesch et al., 2011). After application, the needle was left in place for at least 1-3 min to allow optimal diffusion of lidocaine. Successful lidocaine-induced manipulation was confirmed by reduction of visually evoked responses in V1 (see Fig. 8B).

\section{Retrograde tracer and histology}

Anesthetized rats were immobilized into a preformed mold fixed into the stereotaxic apparatus and received unilateral injections of Fluorogold (FG, Fluorochrome) in S1 (2.4-2.6 $\mathrm{mm}$ posterior to bregma and 5.5-5.8 $\mathrm{mm}$ from the midline). A total volume of $100 \mathrm{nl} \mathrm{FG} \mathrm{(5 \%} \mathrm{in} \mathrm{PBS)} \mathrm{was}$ delivered via a $26 \mathrm{G}$ needle attached to a pump controller. The slow injection speed $(30 \mathrm{nl} / \mathrm{min})$ and the maintenance of the syringe in place for at least 2-3 min ensured an optimal diffusion of the tracer. After a survival time of $4-8 \mathrm{~d}$, the rats were deeply anesthetized with ketamine/ xylazine and perfused transcardially with $4 \%$ PFA. For FG staining, the brains were removed and postfixed in the same solution for $24-72 \mathrm{~h}$. Blocks of tissue containing S1 or V1 were sectioned in the coronal plane at $100 \mu \mathrm{m}$, air dried, and examined using ultraviolet excitation filter. For quantification, FG-stained cells were counted by eye.

For cytochrome oxidase and Nissl staining, the brains were removed and halved along the midline. The subcortical brain regions of one half of the brain were removed, and the cortex was flattened between two acrylic glass plates. Both halves were postfixed in $4 \%$ PFA for $24-72 \mathrm{~h}$. The flattened cortices were sectioned in the transverse plane at $100 \mu \mathrm{m}$ and processed for cytochrome oxidase histochemistry (see Fig. 1B). Briefly, the sections were incubated in a solution containing diaminobenzidine $(0.5 \mathrm{mg} / \mathrm{ml})$, cytochrome C $(0.6 \mathrm{mg} / \mathrm{ml})$, katalase $(0.36 \mathrm{mg} / \mathrm{ml})$, and saccharose $(44.4 \mathrm{mg} / \mathrm{ml})$. The sections were examined using light microscopy and a red (535-555 $\mathrm{nm}$ ) excitation filter of the fluorescence microscope (SZX16, Digital camera DP72, Olympus) to reconstruct the trace of DiI-labeled Michigan probe. The nonflattened brain halves were sectioned in the coronal plane at $100 \mu \mathrm{m}$ and air dried. Fluorescent Nissl staining was performed as previously described (Brockmann et al., 2011) using the NeuroTrace 500/525 green fluorescent Nissl stain (Invitrogen). Briefly, rehydrated slices were incubated for $20 \mathrm{~min}$ with NeuroTrace (dilution 1:100). Sections were washed, coverslipped with Fluoromount and examined using the green $(460-480 \mathrm{~nm})$ and the red $(535-555 \mathrm{~nm})$ excitation filter of the fluorescence microscope. The photographs were adjusted for brightness and contrast using Adobe Photoshop CS4 (Version 11.0.2).

\section{Data analysis and statistics}

Data were imported and analyzed offline using custom-written tools in Matlab software version 7.7 (MathWorks). Data are presented as mean \pm SEM. All values were tested for normal distribution with Lilliefors test $(\alpha=0.05)$. Significance levels of $\left.p<0.05\left(^{*}\right), p<0.01{ }^{* *}\right)$, and $p<$ $\left.0.001{ }^{* * *}\right)$ were detected.

Calculation of evoked potentials. Continuous recordings were epoched offline and 1-s-long time windows (300 ms before stimulus and $700 \mathrm{~ms}$ 
after stimulus) corresponding to each stimulation condition (unimodal visual/tactile, cross-modal congruent/incongruent) were averaged for each recording. Epochs with stimulation artifacts or offsets were cut out. The properties of resulting evoked potentials (EPs) were used to confirm the position of recording sites across rats (maximum amplitude of EPs in layer IV, polarity reversal at the border between layer III and IV). This laminar organization was confirmed by histology (track of DiI-labeled electrode), whereas current source density (CSD) analysis allowed functional identification of $S, G$, and I layers at higher spatial resolution. One-dimensional CSD profiles were calculated to a five point formula (Nicholson and Freeman, 1975). The CSD values $I_{\mathrm{m}}$ were derived from the smoothed second spatial derivative of the extracellular field potentials $\Phi$ and calculated as follows:

$$
\mathrm{I}_{\mathrm{m}}=-\frac{1}{\mathrm{kh}^{2}} \sum_{\mathrm{m}=-\mathrm{n}}^{\mathrm{n}} \mathrm{a}_{\mathrm{m}} \Phi(\mathrm{r}+\mathrm{mh})
$$

where $h$ is the distance between electrodes $(100 \mu \mathrm{m})$ and $r$ is the coordinate perpendicular to the cortical layer $\left(n=2, k=7 a_{0}=-2, a_{ \pm 1}=-1\right.$ and $a_{ \pm 2}=2$ ). The blue in the plots represented current sinks, and red represented current sources. The number of time windows averaged over rats for each recording site was kept constant for each stimulation condition [from recording site 1 (up) to 16 (bottom): 707, 902, 1256, 1264, 1566, 1760, 1754, 1769, 1754, 1755, 1664, 1462, 1567, 1461, 1310, 983]. The EP onset was calculated for each trial as the delay between stimulus and first EP deflection exceeding the baseline SD ( -300 to $0 \mathrm{~ms}$ ). The EP peaks were detected as local local field potential (LFP) maxima/minima within sliding time windows of $100 \mathrm{~ms}$. The peaks were defined as positive $(\mathrm{P})$ or negative $(\mathrm{N})$ based on surface polarity. Their amplitude and delay from stimulus were averaged over all trials and tested together with the EP onsets for normal distribution as well as for significant differences between conditions with Kruskal-Wallis test corrected with the HolmBonferroni method. For scatter plots, the amplitude of P1 and N1 of EPs (see Fig. 2C) was randomized over all trials in all investigated rats and plotted for each layer and stimulation condition. To decide whether the amplitude distribution significantly changes between stimulation conditions, the Euclidean distances between points belonging to different conditions (intercluster Euclidean distances) and Euclidean distances in mixed condition group (intracluster Euclidean distances) were calculated and tested for significance with Kruskal-Wallis test ( $\alpha=0.05$ ). The effect of lidocaine was assessed by calculating the area under the curve for every tactile response in S1 and visual response in V1 under control conditions and after drug application.

Spectral analysis of induced activity. For each stimulation trial, continuous wavelet coefficients $C$ were calculated for time windows of $1500 \mathrm{~ms}$ (500 ms before stimulus and $1000 \mathrm{~ms}$ after stimulus) at frequency scale $a$ and position $b$ by the following:

$$
\mathrm{C}_{\mathrm{a}, \mathrm{b}}=\int_{\mathrm{R}} \Phi(\mathrm{t}) \frac{1}{\sqrt{\mathrm{a}}} \overline{\Psi\left(\frac{\mathrm{t}-\mathrm{b}}{\mathrm{a}}\right)} \mathrm{dt}
$$

where $\Psi$ is a Morlet wavelet. They were corrected for pink noise by normalization to the coefficients of baseline activity (100-300 ms before stimulus) at every frequency. The timing of power modulation is less precise because of the pink noise correction and the low time resolution of wavelets in low-frequency range. Baseline normalized wavelets were averaged for all rats, and their coefficients were tested for significant differences between unimodal and cross-modal stimulation condition by two-sample $t$ test. Significant coefficients were grouped in four time windows and averaged. The amplitude of induced oscillations in different frequency bands was calculated for individual trials (300 ms before stimulus and $700 \mathrm{~ms}$ after stimulus) using the Hilbert transform of filtered data (third-order Butterworth bandpass filter) and averaged for all trials. Mean amplitude of oscillations during time windows before (50$150 \mathrm{~ms})$ and after (75-175 ms; 300-400 ms) stimulus was tested for significant differences with Kruskal-Wallis test.

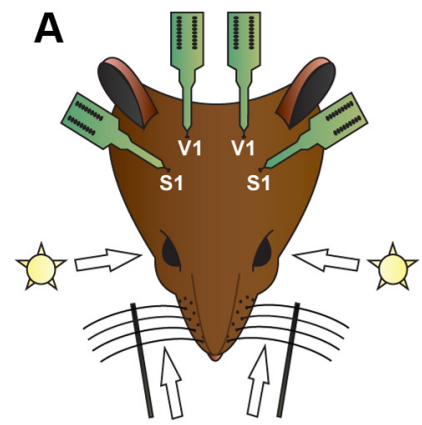

B
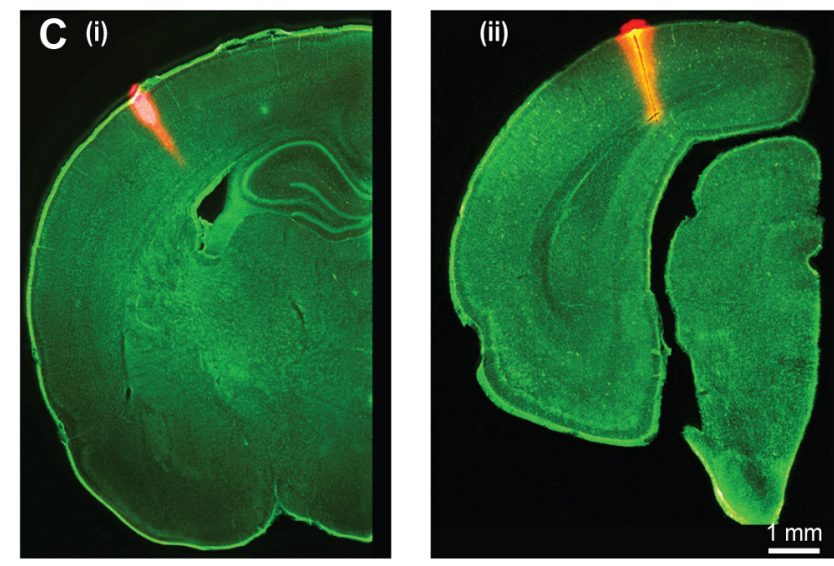

Figure 1. Location of multisite recording electrodes in the barrel field of S1 and in V1 during visual-tactile stimulation protocol. $\boldsymbol{A}$, Schematic drawing displaying sensory stimulation via whisker deflection and/or light flashes as well as the position of multisite Michigan probes in S1 and V1 of Brown Norway rats. $\boldsymbol{B}$, Digital photomontage reconstructing the position of the Dil-labeled electrode (red) in the posteromedial barrel field of cytochrome oxidase-stained flattened sections. In the majority of cases, the recording electrode was centered on a barrel. $\boldsymbol{C}$, Digital photomontage reconstructing the location of the Dil-labeled 16-site recording electrode (orange) in S1 (i) and V1 (ii) of Nissl-stained (green) 100- $\mu$ m-thick coronal sections.

\section{Phase analysis}

Phase distribution across trials was characterized by calculating the resultant length of the mean vector. For this, LFPs during a 1500-ms-long time windows ( $500 \mathrm{~ms}$ before stimulus and $1000 \mathrm{~ms}$ after stimulus) were filtered in three different frequency ranges [third-order Butterworth bandpass filter $(4-12 \mathrm{~Hz}, 13-30 \mathrm{~Hz}, 31-100 \mathrm{~Hz})]$. The phase of oscillatory activity was extracted using the Hilbert transform, and single trial event-related phase values were analyzed by circular statistical methods (Circular Statistic Toolbox). Because of zero-phase digital filtering, the phase was not distorted but the time resolution of phase distribution was poor. The mean resultant vector length was calculated at each frequency and time point and baseline-normalized (300-500 ms before stimulus). The $99 \%$ confidence intervals were calculated with a $z$-value corrected for the number of all time points $(n=1628, z=4.5214)$.

\section{Results}

Evoked potentials in $S 1$ as result of unimodal versus visual-tactile stimulation

We first assessed the impact of unimodal stimulation (light flash or deflection of principal whiskers) on the LFP recorded over the entire cortical depth of the posterior medial barrel subfield (Figures 1 and $2 A$ ) in $S 1$ of lightly urethane-anesthetized Brown Norway rats $(n=10)$. The good visual acuity of pigmented Brown Norway rats compared with albino rats (Prusky et al., 2002) makes them well suited for testing visual-somatosensory processing. By conducting the entire investigation under sleep-like conditions mimicked by the urethane anesthesia (Clement et al., 2008), we avoided the interference with spontaneous whisking 

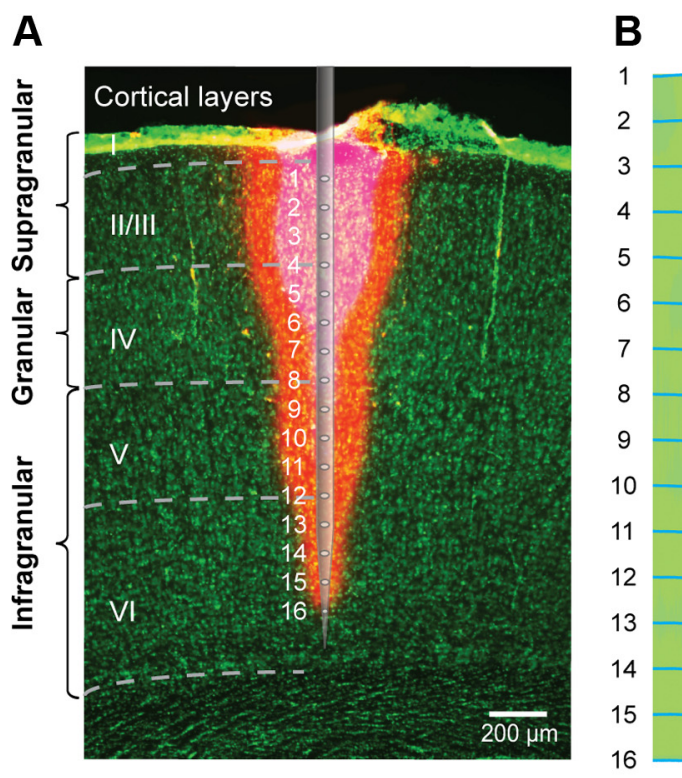

C
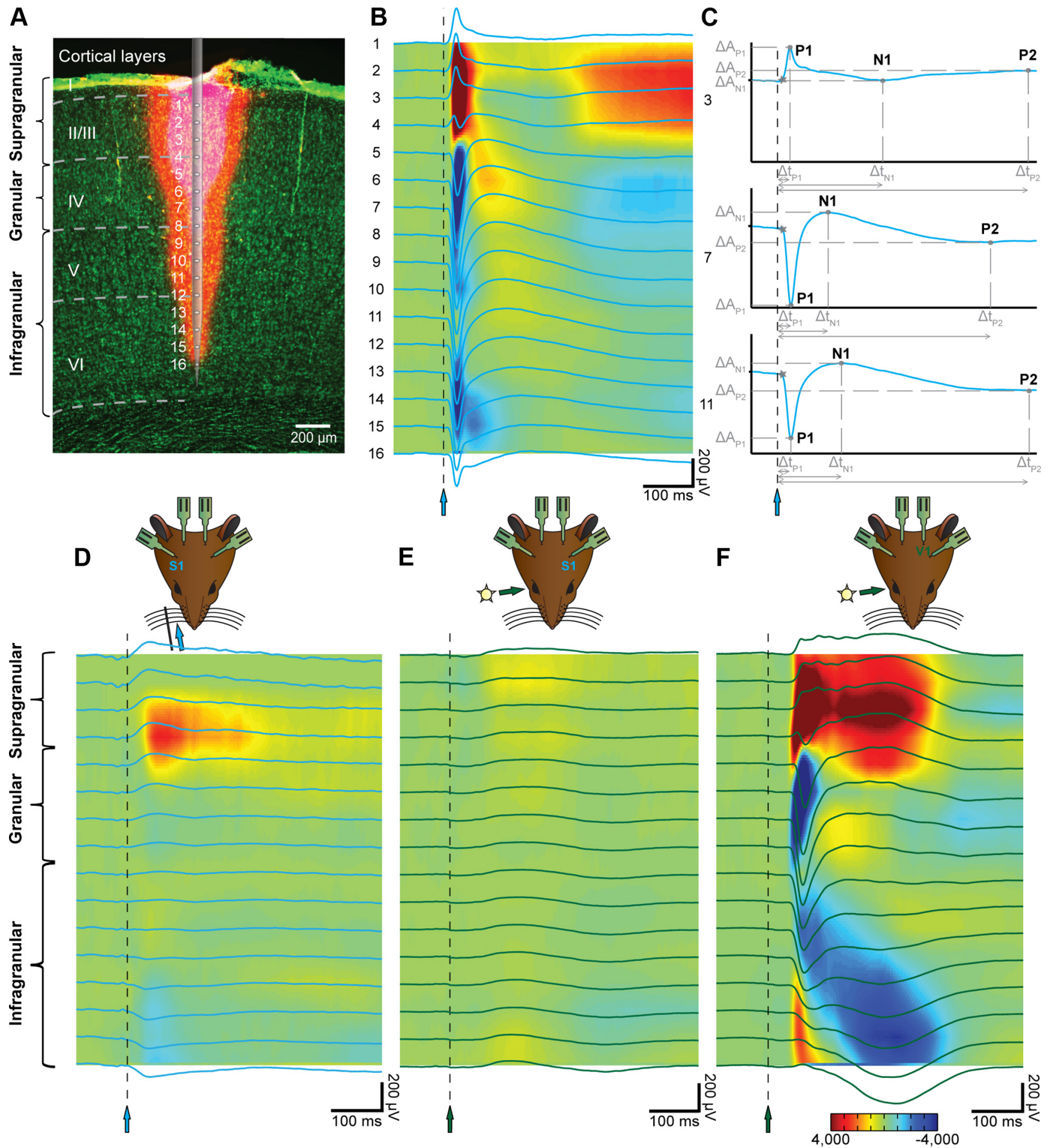

F

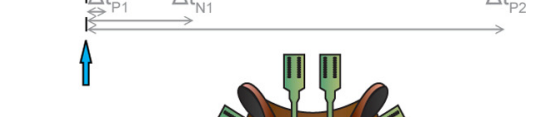


Table 1. Onset and peak time of EPs over S1 depth after unimodal (whisker deflection) and cross-modal stimulation

\begin{tabular}{|c|c|c|c|c|c|c|c|c|}
\hline \multirow[b]{2}{*}{ Electrode position/depth $(\mu \mathrm{m})$} & \multicolumn{4}{|c|}{ Unimodal (tactile stimulation) } & \multicolumn{4}{|c|}{ Bimodal (visual-tactile stimulation) } \\
\hline & Onset (ms) & Peak 1 (ms) & Peak 2 (ms) & Peak 3 (ms) & Onset (ms) & Peak 1 (ms) & Peak 2 (ms) & Peak 3 (ms) \\
\hline $1 / 100$ & $14.0 \pm 1.1(\mathrm{NS})$ & $21.6 \pm 0.3\left(^{* * *}\right)$ & $237.0 \pm 6.0$ (NS) & $447.5 \pm 7.4\left(^{*}\right)$ & $15.1 \pm 1.8$ & $20.8 \pm 0.2$ & $251.6 \pm 7.6$ & $488.3 \pm 6.7$ \\
\hline $2 / 200$ & $15.0 \pm 1.2$ (NS) & $21.3 \pm 0.2$ (NS) & $230.5 \pm 5.4$ (NS) & $470.3 \pm 6.2$ (NS) & $15.1 \pm 2.4$ & $21.8 \pm 0.2$ & $244.1 \pm 6.3$ & $503.4 \pm 5.7$ \\
\hline $4 / 400$ & $18.4 \pm 2.0$ (NS) & $23.5 \pm 0.5$ (NS) & $93.3 \pm 1.6$ (***) $^{*}$ & $369.5 \pm 4.5\left(^{* * *}\right)$ & $20.1 \pm 3.0$ & $26.7 \pm 0.7$ & $120.4 \pm 2.3$ & $404.5 \pm 4.3$ \\
\hline $5 / 500$ & $15.5 \pm 1.4$ (NS) & $29.1 \pm 0.3$ (NS) & $155.1 \pm 2.9$ (***) $^{* *}$ & $\left.414.4 \pm 4.0{ }^{* *}\right)$ & $13.9 \pm 1.1$ & $28.9 \pm 0.3$ & $142.4 \pm 2.3$ & $424.3 \pm 3.6$ \\
\hline $6 / 600$ & $13.8 \pm 1.0$ (NS) & $27.4 \pm 0.2$ (NS) & $157.6 \pm 2.7$ (***) $^{* *}$ & $436.9 \pm 3.7$ (NS) & $12.1 \pm 0.4$ & $27.0 \pm 0.2$ & $134.9 \pm 1.7$ & $430.0 \pm 3.2$ \\
\hline 9/900 & $13.0 \pm 0.6$ (NS) & $27.0 \pm 0.3$ (NS) & $166.0 \pm 2.1$ (***) $^{*}$ & $444.3 \pm 3.7\left(^{* * *}\right)$ & $11.7 \pm 0.3$ & $26.4 \pm 0.2$ & $151.6 \pm 1.5$ & $439.7 \pm 3.4$ \\
\hline $10 / 1000$ & $13.2 \pm 0.6$ (NS) & $27.1 \pm 0.3$ (NS) & $190.1 \pm 2.6\left(^{* * *}\right)$ & $448.9 \pm 3.8\left(^{* * *}\right)$ & $12.9 \pm 0.5$ & $26.7 \pm 0.3$ & $160.8 \pm 1.5$ & $443.4 \pm 3.6$ \\
\hline $11 / 1100$ & $13.8 \pm 1.1$ (NS) & $27.2 \pm 0.3$ (NS) & $203.3 \pm 2.6$ (***) $^{* *}$ & $\left.455.1 \pm 3.9^{(* * *}\right)$ & $12.9 \pm 0.5$ & $27.2 \pm 0.3$ & $172.1 \pm 1.7$ & $452.5 \pm 3.7$ \\
\hline $12 / 1200$ & $13.6 \pm 0.7$ (NS) & $27.7 \pm 0.4$ (NS) & $211.0 \pm 2.8$ (***) $^{* *}$ & $\left.461.2 \pm 4.3^{(* * *}\right)$ & $13.3 \pm 0.7$ & $28.0 \pm 0.4$ & $207.0 \pm 3.0$ & $457.5 \pm 4.2$ \\
\hline $13 / 1300$ & $13.5 \pm 0.8$ (NS) & $29.3 \pm 0.6(* *)$ & $234.5 \pm 3.3$ (***) $^{* *}$ & $464.3 \pm 4.4(* * *)$ & $13.1 \pm 0.6$ & $29.4 \pm 0.5$ & $237.2 \pm 3.7$ & $459.2 \pm 4.3$ \\
\hline $14 / 1400$ & $16.8 \pm 1.9$ (NS) & $33.9 \pm 0.9\left(^{* * *}\right)$ & $244.1 \pm 3.7$ (***) $^{* *}$ & $463.1 \pm 4.7$ (NS) & $17.2 \pm 1.6$ & $34.2 \pm 0.8$ & $261.8 \pm 4.2$ & $457.9 \pm 4.7$ \\
\hline
\end{tabular}

Values are mean \pm SEM for both unimodal and cross-modal stimulation conditions. NS, Not significant.

${ }^{*} p<0.05$ (Kruskal-Wallis test).

${ }^{* *} p<0.01$ (Kruskal-Wallis test).

${ }^{* * *} p<0.001$ (Kruskal-Wallis test).

Table 2. Amplitude of EPs over S1 depth after unimodal (whisker deflection) and cross-modal stimulation

\begin{tabular}{|c|c|c|c|c|c|c|}
\hline \multirow[b]{2}{*}{ Electrode position/depth ( $\mu \mathrm{m})$} & \multicolumn{3}{|c|}{ Unimodal (tactile stimulation) } & \multicolumn{3}{|c|}{ Bimodal (visual-tactile stimulation) } \\
\hline & Peak $1(\mu \mathrm{V})$ & Peak $2(\mu \mathrm{V})$ & Peak $3(\mu \mathrm{V})$ & Peak $1(\mu \mathrm{V})$ & Peak $2(\mu \mathrm{V})$ & Peak $3(\mu \mathrm{V})$ \\
\hline $1 / 100$ & $255.0 \pm 6.5(\mathrm{NS})$ & $26.9 \pm 6.6(*)$ & $66.6 \pm 6.0$ (NS) & $253.4 \pm 6.7$ & $42.1 \pm 6.1$ & $83.8 \pm 6.3$ \\
\hline $3 / 300$ & $228.9 \pm 5.5$ (NS) & $0.8 \pm 5.0$ (NS) & $74.6 \pm 5.3(\mathrm{NS})$ & $214.8 \pm 6.4$ & $7.3 \pm 4.7$ & $87.3 \pm 5.0$ \\
\hline $4 / 400$ & $-18.2 \pm 6.6$ (NS) & $84.5 \pm 3.7\left(^{* * *}\right)$ & $-8.6 \pm 4.7\left(^{* *}\right)$ & $-24.9 \pm 6.2$ & $100.6 \pm 3.3$ & $-18.5 \pm 4.6$ \\
\hline $5 / 500$ & $-313.7 \pm 7.2$ (NS) & $96.2 \pm 2.8\left(^{* * *}\right)$ & $-40.9 \pm 4.0$ (NS) & $-320.8 \pm 6.6$ & $126.0 \pm 2.8$ & $-56.0 \pm 3.8$ \\
\hline $6 / 600$ & $-498.0 \pm 7.2\left({ }^{* *}\right)$ & $104.0 \pm 2.6\left(^{* * *}\right)$ & $-85.5 \pm 3.8\left(^{*}\right)$ & $-515.9 \pm 6.8$ & $136.9 \pm 2.6$ & $-105.2 \pm 3.9$ \\
\hline $9 / 900$ & $-527.4 \pm 6.3\left(^{* * *}\right)$ & $83.8 \pm 2.8\left(^{* * *}\right)$ & $-130.4 \pm 3.96\left(^{* *}\right)$ & $-562.7 \pm 6.3$ & $118.3 \pm 2.5$ & $-144.2 \pm 3.6$ \\
\hline $10 / 1000$ & $-496.5 \pm 5.8\left(^{* * *}\right)$ & $76.6 \pm 2.9\left(^{* * *}\right)$ & $-125.2 \pm 3.7\left(^{*}\right)$ & $-535.3 \pm 5.9$ & $107.0 \pm 2.6$ & $-137.1 \pm 3.5$ \\
\hline $11 / 1100$ & $-464.2 \pm 5.6\left(^{* * *}\right)$ & $61.6 \pm 3.2\left(^{* * *}\right)$ & $-128.5 \pm 3.9$ (NS) & $-493.8 \pm 5.9$ & $94.0 \pm 3.0$ & $-125.4 \pm 3.7$ \\
\hline $12 / 1200$ & $-435.9 \pm 5.9\left(^{* * *}\right)$ & $53.5 \pm 3.5\left(^{* * *}\right)$ & $-126.3 \pm 4.2$ (NS) & $-459.2 \pm 6.0$ & $79.6 \pm 3.5$ & $-111.8 \pm 4.0$ \\
\hline $13 / 1300$ & $-426.1 \pm 6.0\left(^{* * *}\right)$ & $34.0 \pm 3.9\left(^{* * *}\right)$ & $-115.3 \pm 4.1$ (NS) & $-449.2 \pm 6.0$ & $59.8 \pm 3.7$ & $-107.8 \pm 4.2$ \\
\hline $14 / 1400$ & $-400.2 \pm 6.5\left(^{* *}\right)$ & $25.9 \pm 4.6\left(^{* *}\right)$ & $-102.6 \pm 4.7$ (NS) & $-415.7 \pm 6.5$ & $43.7 \pm 4.4$ & $-96.6 \pm 4.8$ \\
\hline
\end{tabular}

Values are mean \pm SEM for both unimodal and cross-modal stimulation conditions. NS, Not significant.

${ }^{*} p<0.05$ (Kruskal-Wallis test).

${ }^{* *} p<0.01$ (Kruskal-Wallis test).

${ }^{* * *} p<0.001$ (Kruskal-Wallis test).

and the impact of alert state, which modulates the cross-modal integration.

In the contralateral S1 whisker, stimulation evoked responses with first fast peaks followed by slower components with positive or negative polarity (Fig. $2 B, C$ ). These mean EPs resulted from averaging the LFPs over a large number (100/rat) of whisker stimulations and had a precisely stimulus-timed onset. For given depths corresponding to the supragranular $(S)$, granular $(G)$ and infragranular (I) layers of the contralateral S1 (Fig. 2B), the EP onset and the delay of the first EP peak did not significantly differ across rats $(\mathrm{S}, p=0.19 ; \mathrm{G}, p=0.19 ; \mathrm{I}, p=0.26)$. Whisker stimulation evoked a smaller response in the ipsilateral S1 (Fig. $2 D$ ), which resulted most likely from the activation of noncrossing projections. Multicomponent stimulus-locked EPs in the contralateral S1 were accompanied by a switch from current sinks to sources at the border between granular and supragranular layers (Fig. 2B). In the granular layer, the EPs had the shortest onset
(12.8 $\pm 0.6 \mathrm{~ms}, n=1769$ unimodal trials) and their first peak showed the largest absolute amplitude $(552.9 \pm 7.1 \mu \mathrm{V}, n=1754$ trials, $p<0.001)$ as well as positive surface polarity (P1). In line with the feedforward activation that has been previously identified in primary cortical areas upon stimulation (Schroeder et al., 1998; Fu et al., 2003), the initial activation of supragranular (onset $16.4 \pm 1 \mathrm{~ms}, n=1032$ trials) and infragranular (onset $14.9 \pm$ $0.5 \mathrm{~ms}, n=1457$ trials $)$ layers significantly $(p<0.001, p=0.001)$ lagged behind the granular response. The initial peak of EPs was followed after a long delay $(\sim 150-400 \mathrm{~ms})$ by two or three additional peaks with positive or negative polarity and lower amplitude (Tables 1 and 2). The most prominent among them had negative surface polarity (N1) and shortest peak time (154 \pm 2.3 ms, $n=1754$ trials) in the granular layer. In contrast to whisker deflection, a short light flash evoked after $73.6 \pm 4.3 \mathrm{~ms}$ a significantly smaller response in the contralateral $S 1$ that had the same polarity over all cortical layers (Fig. 2E). As expected, strong ac- 


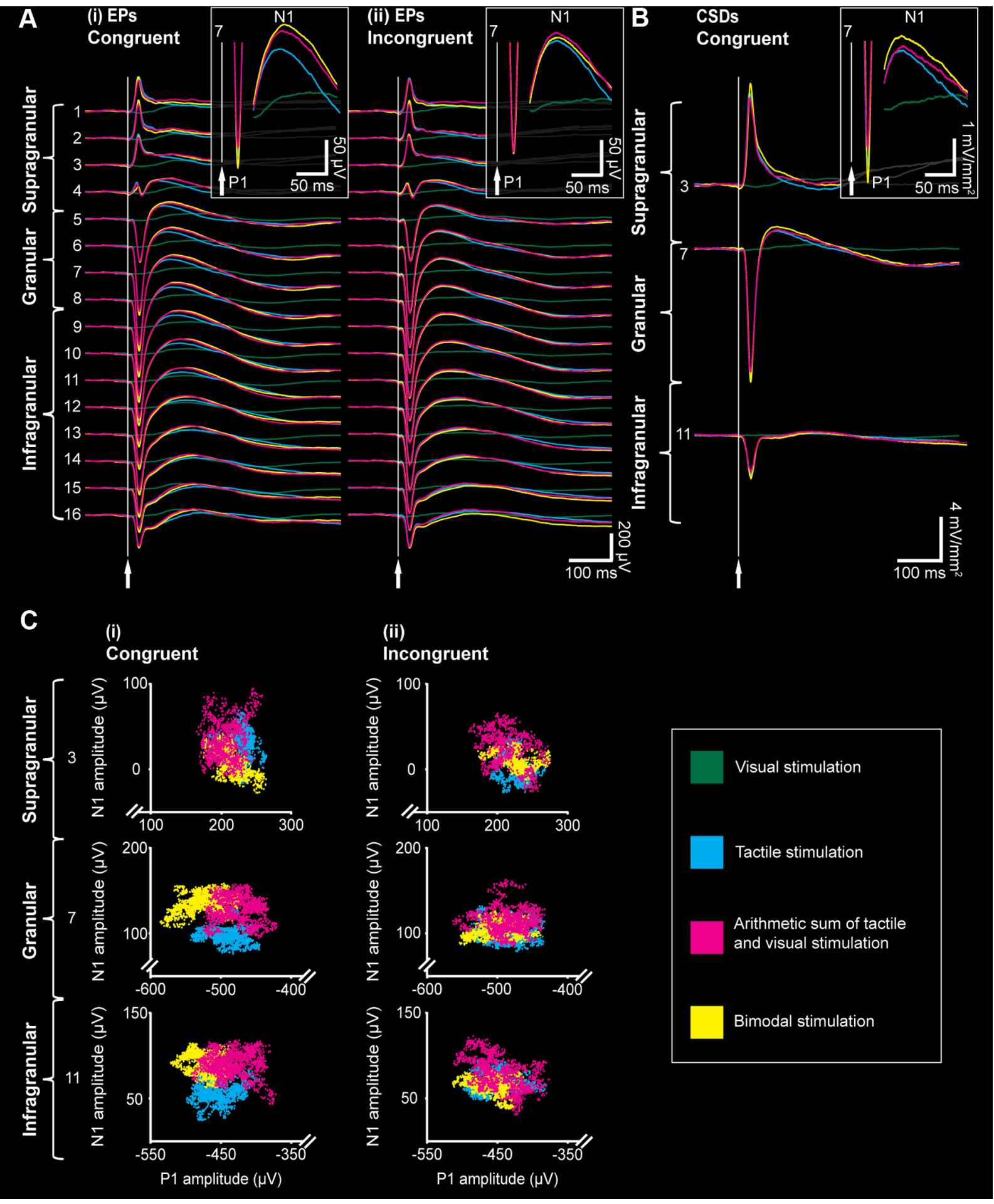

Figure 3. Unimodal versus cross-modal evoked responses in the contralateral barrel field of $S 1$. A, Averaged potentials evoked by visual (green), tactile (cyan), and cross-modal (yellow) stimulation in the supragranular, granular, and infragranular layers of $\mathrm{S} 1$ and compared with the arithmetic sum of the tactile and visual responses (magenta). The bimodal stimulation was performed either congruently (i) or incongruently (ii). Inset, Stimulus-evoked responses in the granular layer displayed at larger magnification. There is the presence of a supra-additive effect at the first positive (P1) and negative (N1) peaks after congruent but not incongruent stimulation. B, Averaged CSD signals after visual (green), tactile (cyan), and congruent cross-modal (yellow) stimulation in the supragranular, granular, and infragranular layers of 1 compared with the arithmetic sum of the tactile and visual responses (magenta). Insets, Supra-additive effect on the P1 and N1 displayed at larger magnification. C, Scatter plots of P1 versus N1 amplitudes for EPs recorded at same location in the supragranular, granular, and infragranular layers of all investigated rats. Congruent (i) but not incongruent (ii) stimulation led to significant ( $p<0.001$ ) disjunction of amplitude clusters for cross-modal stimulation versus unimodal stimulation or arithmetically summed values.

tivation of the contralateral V1 was obtained after lightstimulation (Fig. 2F)

In a second step, whisker deflection and light flashes were presented simultaneously either in the same (congruent) hemi- field or in opposite (incongruent) hemifields with respect to the tactile stimulus (Fig. 3). Compared with the unimodal P1 and N1 $(552.9 \pm 7.1 \mu \mathrm{V}, 98.6 \pm 2.7 \mu \mathrm{V})$ as well as their arithmetic sum $(536.9 \pm 8 \mu \mathrm{V}, 123.5 \pm 3.8 \mu \mathrm{V})$, congruent visual-tactile stimu- 


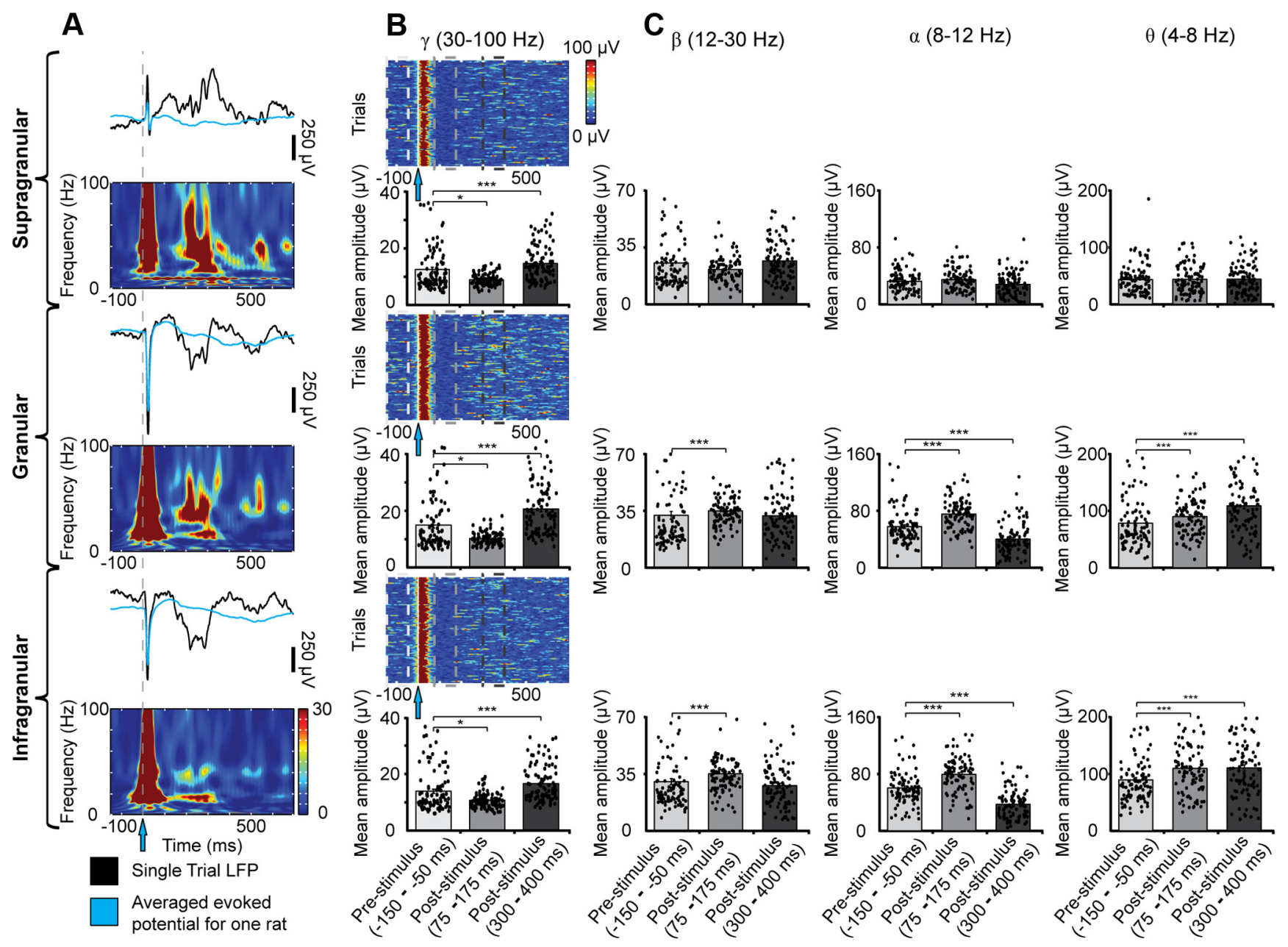

Figure 4. Tactile-induced responses in the contralateral barrel field of S1. A, Single (black) and averaged (cyan) responses to whisker deflection (gray line, cyan arrow) in the supragranular, granular, and infragranular layers of the contralateral S1 accompanied by the corresponding baseline normalized Morlet wavelet spectra for single trials. There is a prominent power increase, especially in $\gamma$ frequency band succeeding the stimulus-evoked response. $\boldsymbol{B}$, Temporal profile of tactile-induced $\gamma$-band oscillations in the supragranular, granular, and infragranular layers from a representative rat. Top, Color-coded plots of $\gamma$ band $(30-100 \mathrm{~Hz})$ amplitude were used for identifying the time windows during which the amplitude of induced oscillations is modified in all trials (dotted medium and dark gray boxes) and comparing them with the prestimulus oscillatory activity (dotted light gray box). Bottom, Bar diagrams (mean \pm SEM) displaying the mean amplitude of $\gamma$ band oscillations during the defined time windows before and after stimulus. Single dots correspond to the amplitudes for each trial. C, Bar diagrams (mean \pm SEM) displaying the amplitude differences for $\beta$ (left), $\alpha$ (middle), and $\theta$ (right) oscillations during the defined time windows after averaging over all stimulation trials. Single dots correspond to amplitudes for each trial. ${ }^{*} p<$ 0.05 (Kruskal-Wallis test). ${ }^{* * *} p<0.001$ (Kruskal-Wallis test).

lation elicited stronger activation of S1 (Fig. 3Ai). Especially the absolute amplitude of both peaks in the granular layer was enhanced $(\mathrm{P} 1,581.8 \pm 6.9 \mu \mathrm{V}, p<0.001 ; \mathrm{N} 1,133.1 \pm 2.5 \mu \mathrm{V}, p<$ $0.001, n=1754$ cross-modal trials). Although the EP onset (11.4 $\pm 0.3 \mathrm{~ms}, n=1769$ trials) and the P1 peak time (26 \pm 0.2 ms, $n=1769$ trials) did not differ between unimodal and crossmodal conditions, the N1 peak time was significantly faster (137.6 $\pm 1.5 \mathrm{~ms}, n=1754, p<0.001)$ after visual-tactile stimulation. By contrast, incongruent bimodal stimulation did not evoke similar supra-additive effects on EPs (Fig. 3Aii). The onset of the cross-modal enhanced first EP peak in the S1 was shorter than the onset of light-evoked response in the contralateral V1 $(37.3 \pm 0.8 \mathrm{~ms}$ ) (Fig. $2 F)$. Thus, visual stimuli augmented the evoked somatosensory response before the activation of V1 by light. The supra-additive effects after bimodal stimulation were not affected by volume conductance and were confirmed by CSD analysis (Fig. 3B). The amplitude of CSD signals for the P1 and $\mathrm{N} 1$ peaks was similarly augmented after bimodal stimulation compared with unimodal stimulation or arithmetic sum of visual and tactile responses. Assessment of intercluster and intracluster
Euclidean distances for the amplitude distributions of the first and second peak for both unimodal and cross-modal evoked EPs confirmed a significant visual-somatosensory enhancement after congruent stimulation only $(\mathrm{S}, p=0.06$; $\mathrm{G}, p<0.001$; I, $p<$ $0.001)$ and the lack of such effects after incongruent activation (Fig. 3C). This enhancement was significant only when tactile and visual stimuli originated in the same hemifield.

\section{Induced oscillatory activity in $S 1$ as result of unimodal versus visual-tactile stimulation}

In addition to prominent multicomponent evoked responses, two additional patterns of network activity characterize S1: (1) spontaneous ongoing oscillations and (2) stimulus-induced oscillations (Senkowski et al., 2008) (Fig. 4A). The first type is known to correlate with various brain states (e.g., up and down states) and has been shown to modulate individual incoming inputs (Civillico and Contreras, 2012). Because they are not causally linked to sensory stimuli, the spontaneous ongoing oscillations are commonly cancelled out after averaging the LFPs corresponding to a large number of stimulation trials. The second type of activity, 


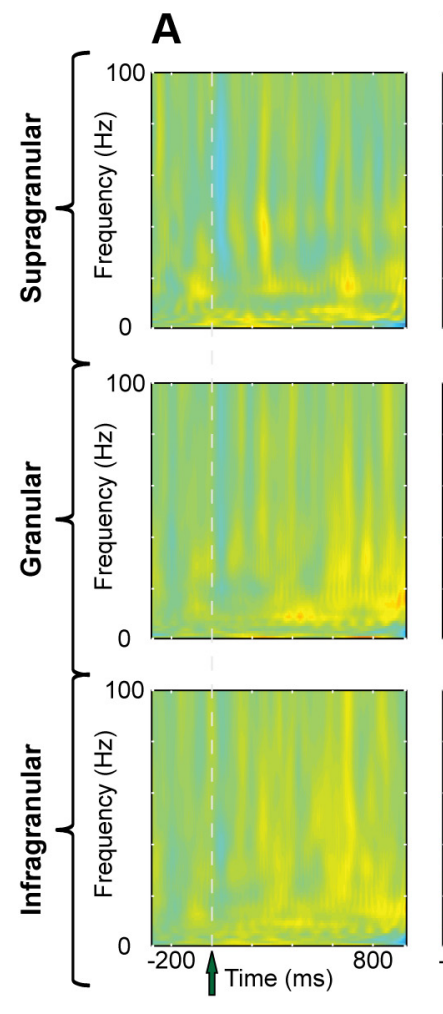

B
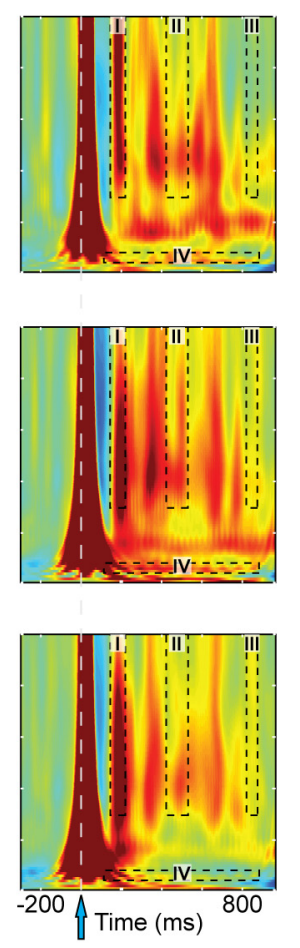

C
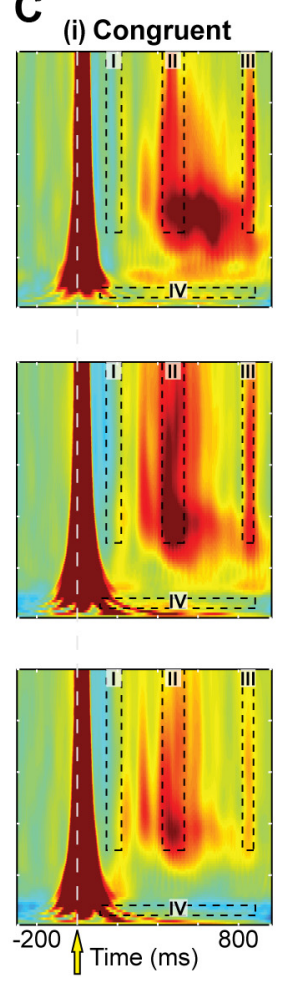

(ii) Incongruent
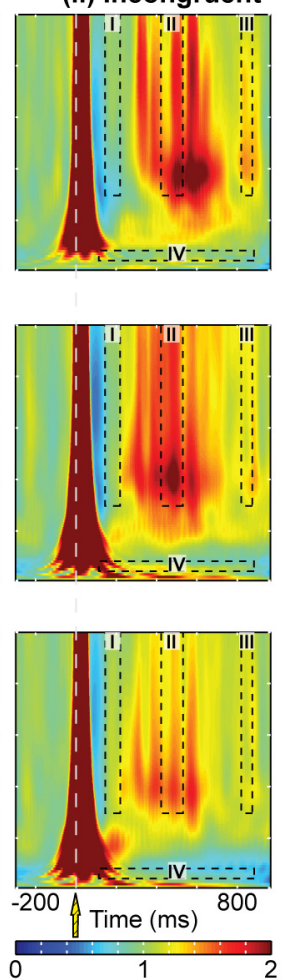

D $\square$ Tactile stimulation
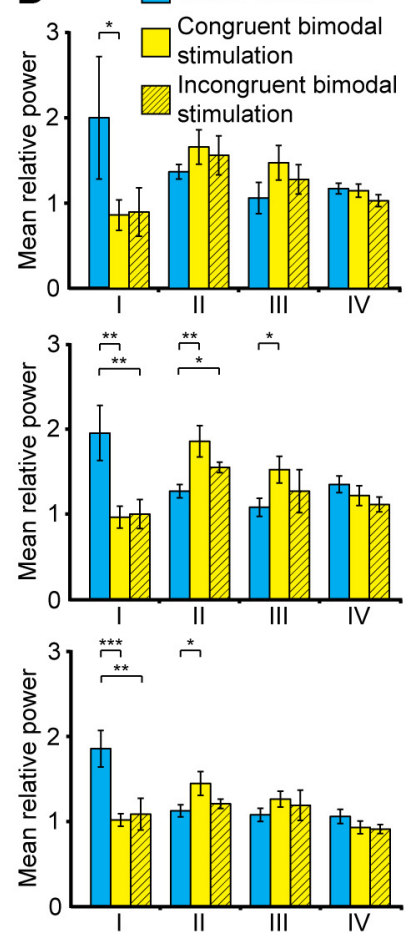

Figure 5. Unimodal and cross-modal induced responses in the contralateral barrel field of S1. Baseline-normalized Morlet wavelet spectra of LFP in S1 supragranular (top), granular (middle), and infragranular (bottom) layers averaged for all rats $250 \mathrm{~ms}$ before and $950 \mathrm{~ms}$ after visual $(\boldsymbol{A})$, tactile $(\boldsymbol{B})$, and congruent $(\boldsymbol{C} \boldsymbol{i})$ and incongruent (Cii) visual-somatosensory stimulation. Stimulus is marked by dotted gray line and arrows. Time windows of activity with significant power changes between tactile and congruent bimodal stimulation are marked by dotted boxes (I-IV). $\boldsymbol{D}$, Bar diagram (mean \pm SEM) displaying the mean power of oscillatory activity during previously defined poststimulus time windows I-IV normalized to the power of activity before stimulus (baseline). ${ }^{*} p<0.05$ (two-sample $t$ test). ${ }^{* *} p<0.01$ (two-sample $t$ test). ${ }^{* * *} p<0.001$ (two-sample $t$ test).

stimulus-induced oscillations, is causally related, but in contrast to the evoked response, not phase-locked to sensory stimulus (e.g., whisker deflection). Consequently, these oscillations are underscored by plotting individual frequency-power spectra for a large number of trials, whereas they cancel out when LFPs were averaged across trials.

Whisker stimulation-induced prominent oscillations with frequencies ranging from $\theta(4-8 \mathrm{~Hz})$ to $\gamma$ band $(30-100 \mathrm{~Hz})$ in the supragranular, granular, and infragranular layers of S1 (Fig. 4). Analysis of individual frequency spectra corresponding to a large number of stimulation trials revealed that, shortly after the prominent stimulus-evoked response, the amplitude of stimulus-induced $\gamma$ band oscillations was significantly $(\mathrm{S}, p=0.02 ; \mathrm{G}, p=0.03 ; \mathrm{I}, p=$ $0.03)$ decreased from $12.6 \pm 0.8 \mu \mathrm{V}(\mathrm{S}), 15 \pm 1 \mu \mathrm{V}(\mathrm{G})$, and $14.1 \pm$ $0.7 \mu \mathrm{V}$ (I) under baseline conditions (50-150 ms before stimulus) to $8.9 \pm 0.2 \mu \mathrm{V}(\mathrm{S}), 10.3 \pm 0.2 \mu \mathrm{V}(\mathrm{G})$, and $10.9 \pm 0.3 \mu \mathrm{V}$ (I) for 75-175 ms time epoch after stimulus. This depression was followed (300-400 ms after stimulus) by a stimulus-induced increase of $\gamma$ band network activity to $14.8 \pm 0.6 \mu \mathrm{V}(\mathrm{S}), 20.7 \pm 1 \mu \mathrm{V}(\mathrm{G})$, and $16.9 \pm 0.6 \mu \mathrm{V}$ (I) that significantly $(p<0.001)$ exceeded the baseline level (Fig. $4 B$ ). Although less consistent across layers, tactile stimulation additionally induced low-frequency oscillations. The amplitude of $\theta$ band activity significantly $(p<0.001)$ increased from $77.9 \pm 4.5$ $\mu \mathrm{V}(\mathrm{G})$ and $89.2 \pm 4.1 \mu \mathrm{V}$ (I) before the stimulus to $89.4 \pm 3.4 \mu \mathrm{V}$ (G) and 109.4 $\pm 4.4 \mu \mathrm{V}$ (I) after the evoked response. Similarly, $\alpha$ and $\beta$ band oscillations were significantly augmented after stimulus (Fig. 4C).

To assess the effect of simultaneous visual and tactile stimulation on the induced oscillatory activity in the S1, we pooled the multiple-trial baseline normalized Morlet wavelet spectra across animals $(n=8)$, separately for the four different stimulation conditions: unimodal visual stimulation, unimodal tactile stimulation, simultaneous congruent cross-modal stimulation, and simultaneous incongruent cross-modal stimulation. Unimodal visual stimulation did not modify the frequency distribution over S1 layers (Fig. 5A). In contrast, whisker deflection induced prominent responses in different frequency bands in the $S, G$, and I layers (Fig. 5B). During cross-modal stimulation, the somatosensory activity was visually modulated. To quantify the effects of cross-modal stimulation on the oscillatory activity, we compared the baseline-normalized power change for each time-frequency point of the Morlet wavelet spectra. The time points with significant power change after cross-modal versus unimodal stimulation were clustered. Three time windows (I: 150-230 ms; II: 440-550 ms; III: 850-900 ms after stimulus) with significantly different induced $\gamma$ band $(30-100 \mathrm{~Hz})$ activity and one time window (IV: 115-915 ms after stimulus) with significantly different induced $\theta$ band $(4-8 \mathrm{~Hz})$ activity resulted after clustering analysis (Fig. $5 B, C$ ). During time window I the mean power relative to baseline decreased in all cortical layers, whereas during time window II the mean power increased after bimodal stimulation. The relative augmentation in $\gamma$ frequency band (defined as fraction with respect to baseline) was more prominent in the granular (from $1.3 \pm 0.1$ to $1.9 \pm 0.2, p=0.004$ ) than in the infragranular and supragranular layers (from $1.1 \pm 0.1$ to $1.5 \pm 0.1, p=0.029$; from $1.4 \pm 0.1$ to $1.7 \pm 0.2, p=0.134)$. Compared with unimodal stimulation, the bimodal stimulus significantly $(p=0.02)$ enhanced the oscillatory activity in the granular layer during time 


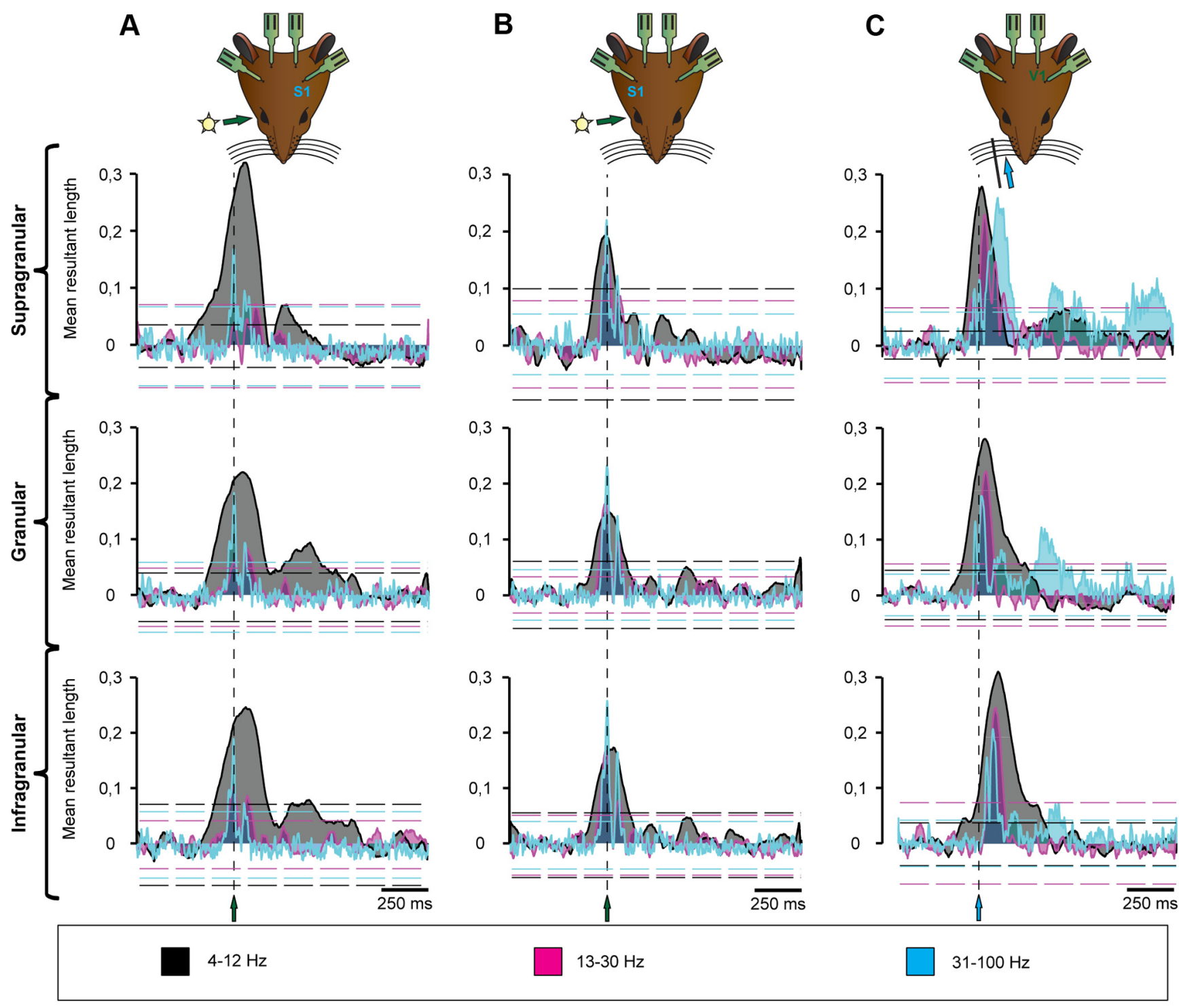

Figure 6. Phase concentration of oscillatory activity after contralateral and ipsilateral stimulation. $A$, Plots displaying the mean resultant vector length of oscillatory phases in $S 1$ after contralateral visual stimulation. The values were averaged over time and layers in all rats for three frequency bands: $\theta-\alpha(4-12 \mathrm{~Hz})$ in black; $\beta(13-30 \mathrm{~Hz})$ in magenta; $\gamma(30-100 \mathrm{~Hz})$ in cyan. $\boldsymbol{B}$, Plots displaying the mean resultant vector length of oscillatory phases in $\mathrm{S} 1$ after ipsilateral visual stimulation. There is less pronounced and shorter phase concentration after ipsilateral stimulation compared with contralateral stimulation. C, Phase concentration of oscillatory activity in V1 after contralateral tactile stimulation. For all plots, the stimulus was marked by dotted black line and arrow. The dashed lines mark the borders of confidence interval for $\alpha=0.01$.

window III $(1.1 \pm 0.1$ to $1.5 \pm 0.2)$. Incongruent visual-tactile stimulation similarly modified the power of induced oscillations during these time windows. However, the changes were less prominent compared with the congruent condition (Fig. 5C,D). The most obvious difference was the decrease in $\gamma$ power of induced oscillations during time window III from $1.5 \pm 0.2$ after congruent stimulation to $1.3 \pm 0.3$ after incongruent stimulation. Thus, visual input accompanying whisker deflection modified the power of induced network oscillations in S1.

\section{Mechanisms and anatomical substrate of visual-tactile interplay}

Next, we aimed at identifying the neural mechanisms by which the copresented visual input modulates the tactile evoked response on one hand and the induced network activity in $\mathrm{S} 1$ on the other hand. This will enable to link both major cross-modal effects into an unitary concept of visual-tactile processing. One possible mechanism is a visual stimulus-induced phase reset of the ongoing network oscillations in S1 to an optimal phase, during which the incoming tactile stimulus has the highest effectiveness. To test this hypothesis, we compared the phase synchrony of spontaneous oscillations in S1 during a large number of unimodal visual stimulation trials and calculated the mean resultant vector length of oscillatory phases for $\theta(4-8 \mathrm{~Hz}), \alpha(8-12 \mathrm{~Hz}), \beta$ $(13-30 \mathrm{~Hz})$, and $\gamma(30-100 \mathrm{~Hz})$ frequencies. If the oscillatory phase is the same in each trial, the mean resultant vector length will be 1 , whereas if the oscillatory phase is absolutely random, the value will be 0 . Unimodal visual stimulation induced prominent, but similar, stimulus-related phase concentration of $\theta$ and $\alpha$ band oscillations in all layers of the contralateral S1. To avoid errors of narrow band filtering and improve the power of statistical testing, the data for $\theta$ and $\alpha$ frequency bands were pooled (Fig. 6A). A similar, but smaller, phase concentration was observed in the ipsilateral S1 (Fig. 6B). Visually driven oscillations in the contralateral S1 remained in phase for a long time, their synchronization being significant (i.e., $>99 \%$ confi- 

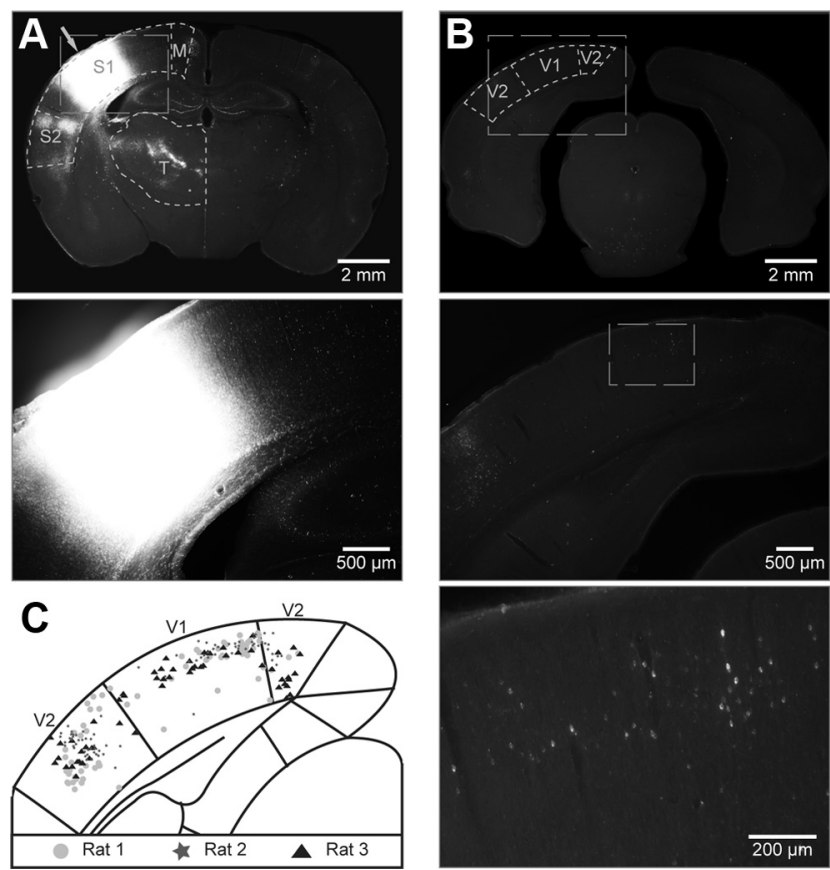

Figure 7. Direct connectivity between $\mathrm{S} 1$ and $\mathrm{V} 1$ revealed by retrograde tracing with fluorogold. $A$, Top, FG spreading from the injection site (arrow) over all layers of $S 1$ in a $100-\mu \mathrm{m}-$ thick coronal slice. There is high density of retrogradely labeled cells in thalamic nuclei (T), motor cortex (M), and secondary somatosensory cortex (S2). Bottom, High-magnification photomicrograph of the injection site marked (dashed box) confirms the restriction of tracer to S1. $\boldsymbol{B}$, Increasing magnification (from top to bottom) retrogradely labeled neurons in V1 and V2. There is low density of stained neurons in V1 compared with V2. C, Number and position of retrogradely labeled neurons in V1 and V2 of three rats (stars, circles, triangles). Most of stained neurons are located in the granular and supragranular layers of $\mathrm{V} 1$.

dence interval) for up to $330 \mathrm{~ms}$ in S, up to $530 \mathrm{~ms}$ in $\mathrm{G}$, and up to $410 \mathrm{~ms}$ in I. Similar cross-modal phase reset for oscillatory activity in $\theta$ and $\alpha$ band was observed in V1 after tactile stimulation (Fig. 6C).

To decide whether the visual-somatosensory interplay relies on direct communication between neuronal networks in V1 and $\mathrm{S} 1$, we first assessed by anatomical tracing the direct connectivity between the two primary sensory cortices. We injected small amounts of the retrograde tracer FG, which has high resistance to fading (Schmued and Fallon, 1986), into S1 of seven rats taking special attention to the confinement of tracer within cortical layers (Fig. 7A). Confirming previous studies, bright fluorescent back-labeling of parent cell bodies feedforwardly projecting to the barrel field was observed in the ventral posteromedial (VPM) and posterior nuclei of thalamus as well as in the ipsilateral secondary somatosensory cortex. Moreover, we identified labeled cells in the visual cortices and to a weaker extent in the motor cortex (Fig. $7 A, B$ ). Remarkably, FG injection revealed that V1 neurons directly project to $S 1$. Retrogradely stained neurons were detected in $\mathrm{V} 1$ of all investigated rats (Fig. $7 B, C$ ), being concentrated in a small cortical volume $\left(0.064 \mathrm{~mm}^{3}\right)$ over the $S$ and $G$ layers. The maximal cell density strongly varied between animals (13-56 cells; mean $28.9 \pm 5.8$ cells), possibly reflecting the slightly variable position of the FG injection site in S1. No stained neurons were observed in the contralateral V1, indicating that direct interhemispheric connections between V1 and S1 are missing. We hypothesize that the connectivity directly linking the primary cortices may represent one of the anatomical substrates underlying functional communication between S1 and V1.
A

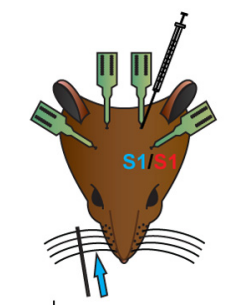

B
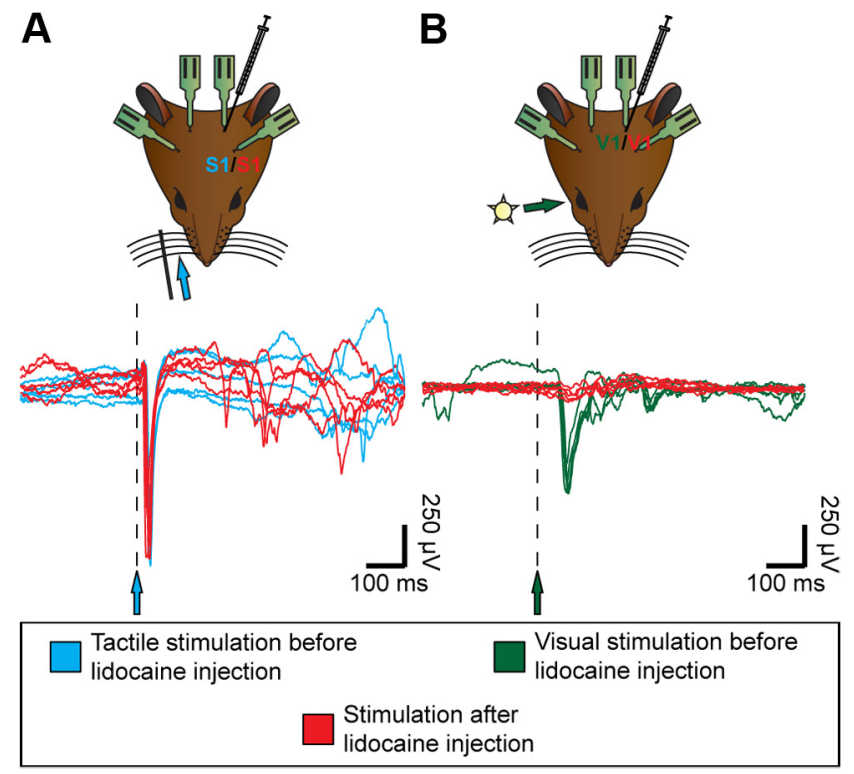

Figure 8. Lidocaine effects on the unimodal evoked and induced responses in S1 and V1. A, Top, Schematic diagram of the experimental protocol depicting the type of unimodal stimulation (whisker deflection), the recording area (S1), and the site of lidocaine injection (V1). Bottom, Superimposed LFP responses to tactile stimulation recorded in the contralateral $S 1$ before (cyan) and after (red) lidocaine injection. The evoked and induced activity is unaffected. $\boldsymbol{B}$, Top, Schematic diagram of the experimental protocol depicting the type of unimodal stimulation (light), recording area (V1), and the site of lidocaine injection (V1). Bottom, Superimposed LFP responses to visual stimulation recorded in the contralateral V1 before (cyan) and after (red) lidocaine injection. There is a decrease of both evoked and induced activity. $\boldsymbol{A}, \boldsymbol{B}$, The dotted lines and arrows indicate the tactile/visual stimulus.

To test this hypothesis, we silenced the electrical activity in V1 ( $n=5$ rats). Small amounts $(100-300 \mathrm{nl})$ of the action potential blocker lidocaine were injected at low speed (200 nl/min) into V1. Confirming our previous data (Brockmann et al., 2011) and in line with the spherical volume calculation, lidocaine acted on a small volume (radius $\sim 290-425 \mu \mathrm{m}$ ) confined to $\mathrm{V} 1$, leading here to a significant reduction $(49.5 \pm 11.4 \%, p<0.001)$ of the visual responses while leaving the tactile responses in S1 unaffected (Fig. 8). The effects of transient and partial V1 silencing were investigated in the ipsilateral barrel cortex. Lidocaine-induced blockade of activity in V1 did not affect the bimodal enhancement of the first EP peak (P1) over the S, G, and I layers (Fig. 9A; Tables 3 and 4). However, lidocaine eliminated the cross-modal augmentation of the second peak (N1), the amplitude of which did not anymore differ between unimodal (S: $25.1 \pm 20.4 \mu \mathrm{V}, n=102$ trials; G: $75.8 \pm 7.3 \mu \mathrm{V}, n=$ 243 trials; I: $56.4 \pm 8.2 \mu \mathrm{V}, n=248$ trials) and cross-modal (S: $21.4 \pm 32.1 \mu \mathrm{V}$; G: $89.4 \pm 9.5 \mu \mathrm{V} ; \mathrm{I}: 46.5 \pm 9.2 \mu \mathrm{V}$ ) stimulation (Fig. $9 A, B)$. Moreover, blockade of V1 activity eliminated almost all power differences between the network activity induced by unimodal versus cross-modal stimulation (Fig. 9C). Partial silencing of V1 allowed also testing whether phase reset of ongoing oscillatory activity mediates the visual-tactile interplay. The analysis of the mean resultant vector length showed that phase concentration of slow oscillations $(4-12 \mathrm{~Hz}$ ) by contralateral visual input was significantly decreased in all $\mathrm{S} 1$ layers and almost abolished in the $\mathrm{S}$ after lidocaine injection into V1 (Fig. 9D). Moreover, functional disconnection of $S 1$ and $V 1$ delayed the remaining phase concentration in the $\mathrm{G}$ and $\mathrm{I}$ ( $\sim 190 \mathrm{~ms}$ and $\sim 180 \mathrm{~ms}$ after stimulus, respectively).

These results indicate that V1 and S1 connections are necessary for cross-modal processing. The direct connectivity demonstrated in the present study might represent one possible pathway 

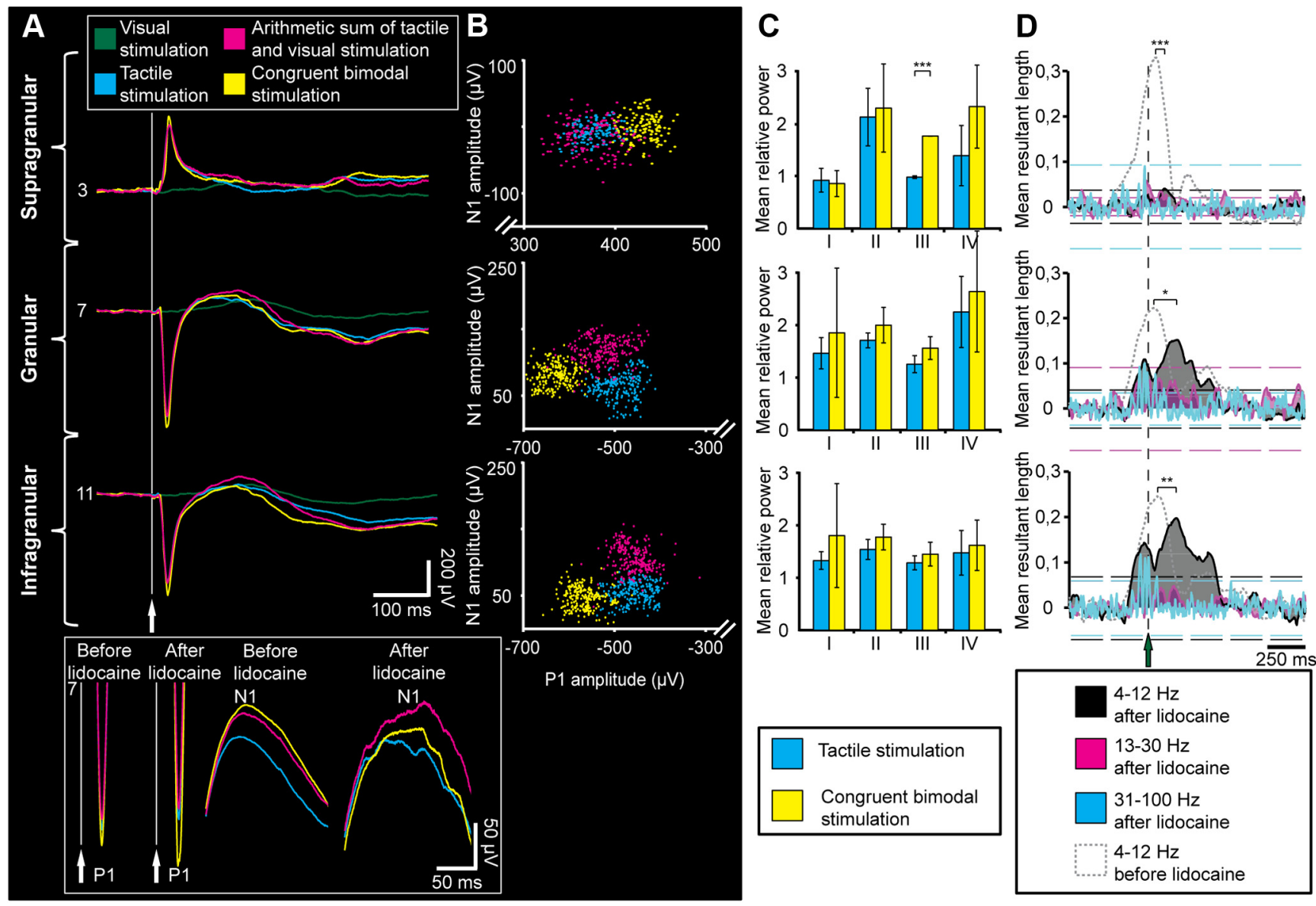

Figure 9. Consequences of lidocaine-induced blockade of $\mathrm{V} 1$ activity on cross-modal responses in S1.A, Averaged potentials evoked by visual (green), tactile (cyan), and congruent cross-modal (yellow) stimulation in the presence of lidocaine in the supragranular, granular, and infragranular layers of $\mathrm{S1}$ compared with the arithmetic sum of the tactile and visual responses (magenta). Inset, Stimulus-evoked responses in the granular layer displayed before and after lidocaine at larger magnification. There is persistence of the supra-additive effect at P1 and its abolishment at N1 after lidocaine injection. B, Scatter plots of $\mathrm{P} 1$ versus $\mathrm{N} 1$ amplitudes for congruent EPs recorded at the same location in the supragranular, granular, and infragranular layers of all investigated rats after lidocaine injection. C, Bar diagram (mean \pm SEM) displaying the power of oscillatory activity induced by tactile (cyan) or congruent bimodal (yellow) stimulation after lidocaine injection. Values were normalized to the power of activity before stimulus (baseline). ${ }^{* * *} p<0.001$ (two-sample $t$ test). $\boldsymbol{D}$, Lidocaine effect on phase concentration of oscillatory activity in $\mathbf{S 1}$ after contralateral visual stimulation. Plots displaying the mean resultant vector length of oscillatory phases after lidocaine blockade. The values were averaged over time for each $S 1$ layer ( $n=5$ rats) in three frequency bands: $\theta-\alpha(4-12 \mathrm{~Hz})$ in black; $\beta(13-30 \mathrm{~Hz})$ in magenta; $\gamma(30-100 \mathrm{~Hz})$ in cyan. The phase concentration in the $4-12 \mathrm{~Hz}$ band before lidocaine blockade is displayed as a dotted gray line. The stimulus was marked by dotted black line and green arrow. The dashed lines indicate the borders of confidence interval for $\alpha=0.01$.

Table 3. Onset and peak time of EPs over S1 depth after unimodal (whisker deflection) and cross-modal stimulation in the presence of lidocaine

\begin{tabular}{|c|c|c|c|c|c|c|c|c|}
\hline \multirow[b]{2}{*}{ Electrode position/depth $(\mu \mathrm{m})$} & \multicolumn{4}{|c|}{ Unimodal (tactile stimulation) } & \multicolumn{4}{|c|}{ Bimodal (visual-tactile stimulation) } \\
\hline & Onset (ms) & Peak 1 (ms) & Peak 2 (ms) & Peak 3 (ms) & Onset (ms) & Peak 1 (ms) & Peak 2 (ms) & Peak 3 (ms) \\
\hline $3 / 300$ & $8.0 \pm 2.0$ (NS) & $28.4 \pm 0.7$ (NS) & $193.9 \pm 25.6\left(^{*}\right)$ & $475.0 \pm 16.3$ (NS) & $8.1 \pm 4.0$ & $28.5 \pm 0.8$ & $89.3 \pm 17.5$ & $472.3 \pm 14.5$ \\
\hline $7 / 700$ & $15.3 \pm 3.7(\mathrm{NS})$ & $27.7 \pm 0.5$ (NS) & $67.0 \pm 3.0\left(^{* *}\right)$ & $344.7 \pm 5.9$ (NS) & $12.9 \pm 2.7$ & $29.8 \pm 1.1$ & $110.1 \pm 3.6$ & $427.9 \pm 10.1$ \\
\hline $11 / 1100$ & $13.0 \pm 2.6$ (NS) & $32.6 \pm 1.6$ (NS) & $79.7 \pm 4.6$ (NS) & $410.3 \pm 8.4$ (NS) & $14.1 \pm 3.5$ & $30.6 \pm 1.1$ & $92.0 \pm 4.4$ & $358.4 \pm 6.5$ \\
\hline
\end{tabular}

Values are mean \pm SEM for both unimodal and cross-modal stimulation conditions. NS, Not significant.

${ }^{*} p<0.01$ (Kruskal-Wallis test).

${ }^{* *} p<0.001$ (Kruskal-Wallis test).

that mediates the visual reset of oscillatory phase in the barrel field, whereas subcortical feedforward interactions seem to account for the supra-additive early evoked responses.

\section{Discussion}

The present study aimed at elucidating the functional and structural correlates of visual-somatosensory interactions by combining electrophysiological recordings and pharmacology in vivo with anatomical tracing. We demonstrate the following: (1) integration of visual and tactile information takes place in the barrel field of S1; (2) visual cross-modal supra-additive augmentation of somatosensory-evoked responses mainly originates on the subcortical sensory tract partially independent of the primary neocortices; and (3) visual stimulus-induced reset of ongoing neuronal oscillations and power modulation of the activity in S1 critically depend on the communication between primary sensory cortices (Fig. 10).

As previously reported (Roy et al., 2011), tactile information from the whisker reached the granular layer of the barrel field after a short delay $(\sim 12 \mathrm{~ms})$. From here, the activity spread to the infragranular and supragranular layers. In addition to evoking such prominent response, the unimodal somatosensory stimulus 
Table 4. Amplitude of EPs over S1 depth after unimodal (whisker deflection) and cross-modal stimulation in the presence of lidocaine

\begin{tabular}{|c|c|c|c|c|c|c|}
\hline \multirow[b]{2}{*}{ Electrode position/depth $(\mu \mathrm{m})$} & \multicolumn{3}{|c|}{ Unimodal (tactile stimulation) } & \multicolumn{3}{|c|}{ Bimodal (visual-tactile stimulation) } \\
\hline & Peak $1(\mu \mathrm{V})$ & Peak $2(\mu \mathrm{V})$ & Peak $3(\mu \mathrm{V})$ & Peak $1(\mu \mathrm{V})$ & Peak $2(\mu \mathrm{V})$ & Peak $3(\mu \mathrm{V})$ \\
\hline $3 / 300$ & $378.9 \pm 17.9\left(^{* *}\right)$ & $25.1 \pm 20.4$ (NS) & $127.3 \pm 20.7$ (NS) & $428.3 \pm 19.3$ & $21.4 \pm 32.1$ & $102.2 \pm 26.8$ \\
\hline $7 / 700$ & $-607.6 \pm 22.7\left(^{*}\right)$ & $75.8 \pm 7.3$ (NS) & $-162.8 \pm 11.5$ (NS) & $-669.5 \pm 18.6$ & $89.4 \pm 9.5$ & $-185.9 \pm 10.4$ \\
\hline $11 / 1100$ & $-506.7 \pm 19.6\left(^{* *}\right)$ & $56.4 \pm 8.2$ (NS) & $-162.5 \pm 10.6\left(^{* *}\right)$ & $-582.7 \pm 18.8$ & $46.5 \pm 9.2$ & $-207.7 \pm 9.6$ \\
\hline
\end{tabular}

Values are mean \pm SEM for both unimodal and cross-modal stimulation conditions. NS, Not significant.

${ }^{*} p<0.05$ (Kruskal-Wallis test).

${ }^{* *} p<0.01$ (Kruskal-Wallis test).

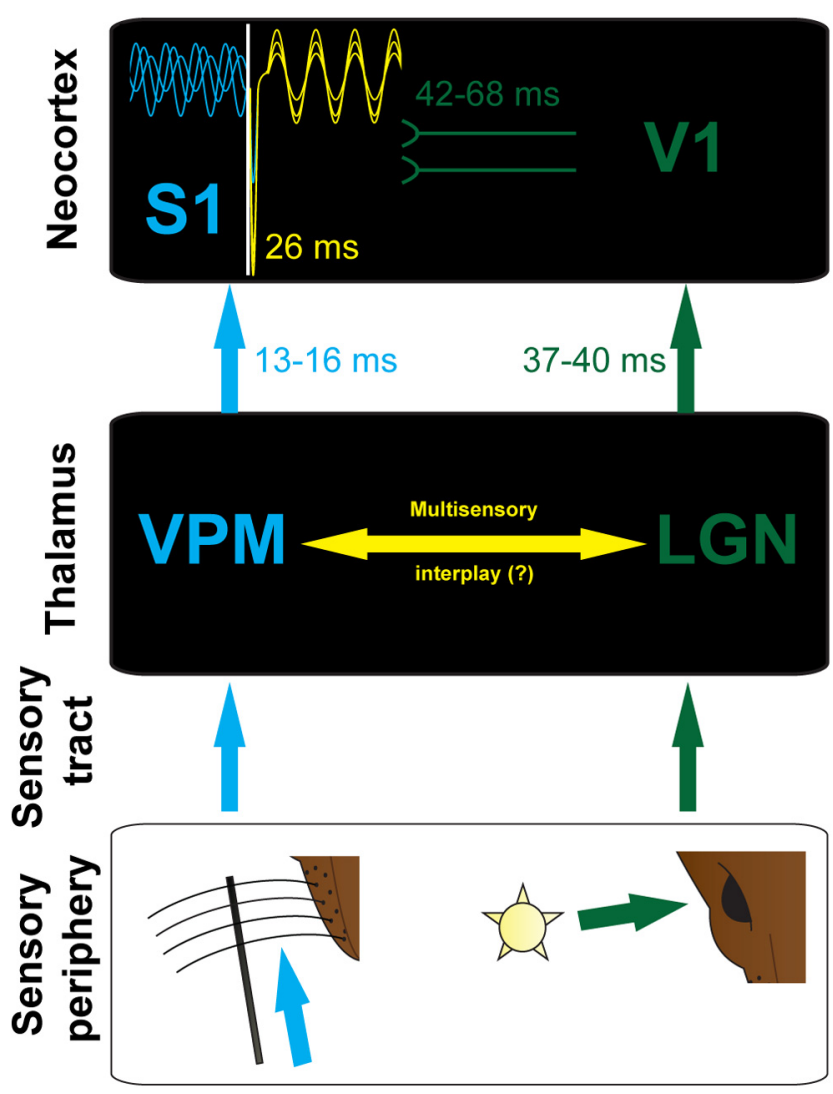

Figure 10. Schematic diagram of the mechanisms and anatomical substrate of visualsomatosensory interplay (yellow) at neocortical and subcortical level. We propose that cooccurring visual (green) and tactile (cyan) stimuli traveling along the sensory tract may be integrated at thalamic and neocortical level. Direct projections from $\mathrm{V} 1$ may reset the phase of network oscillations in S1, whereas cross-modal interactions between VPM, LGN, and other thalamic nuclei might account for augmentation of tactile stimulus-evoked response. Transmission delays are marked on the arrows.

additionally modified S1 network activity and induced neuronal oscillations in a wide range of frequency bands $(4-100 \mathrm{~Hz})$. Simultaneous cross-modal stimulation of the whiskers and the eye augmented the evoked responses and significantly changed the poststimulus power of induced network oscillations in the $\theta$ and $\gamma$ frequency band while leaving the temporal pattern of activation across layers unaffected.

Our results point toward two distinct mechanisms of crossmodal interaction. First, subcortical multisensory regions along the sensory tract that relay information from the periphery to the neocortex may account for cross-modal augmentation of the short-delay initial response in S1. Several lines of evidence support this conclusion. Partial silencing of V1 by lidocaine did not affect the first peak of the evoked response in the S1, suggesting that this processing pathway bypasses V1. Moreover, the first multisensory effect had a shorter latency than the first visual response in both $\mathrm{V} 1$ and $\mathrm{S} 1$. Thalamic nuclei [e.g., lateral geniculate nucleus (LGN), VPM] relay sensory information from the periphery (e.g., retina, whiskers) first to the $G$ layer and later to the $S$ and I layers of the corresponding primary sensory cortices (e.g., V1, S1) (Wise and Jones, 1978; Herkenham, 1980; Chapin and Lin, 1990). In addition to unisensory processing, some thalamic nuclei might mediate multisensory interactions. For this, four distinct mechanisms have been postulated (Cappe et al., 2009): (1) thalamic nuclei send sensory information to more than one sensory cortex; (2) cross-modal input is integrated at the thalamic level and subsequently sent to sensory cortices; (3) sensory cortices (and possibly single neurons) receive input from several thalamic regions processing different sensory inputs; and (4) inputs of one sensory area are transferred to another cortical area via the thalamus (corticothalamo-cortical route) (Noesselt et al., 2010). Because of the limited temporal resolution of imaging techniques (Barth et al., 1995; Noesselt et al., 2010), it was not possible to distinguish whether thalamic multisensory effects are mediated via feedback connections from cortical areas (mechanism 4) or whether they are the result of bottom-up processing (mechanisms 1-3). Our results clearly demonstrate the existence of thalamic feedforward mechanisms because the supra-additive effect on the first peak of the evoked response was not affected by lidocaine and its peak time was too short to allow for feedback interactions. Because the LGN was not retrogradely stained, mechanism 2 is the most probable explanation for the present experimental findings. Several thalamic regions have been reported to react to stimulation of more than one sensory modality, representing possible relay stations of cross-modal effects (Tyll et al., 2011). Simultaneous visual and tactile stimulation activated neurons in the medial geniculate body (Wepsic, 1966) and reticular nucleus (Sugitani, 1979). Similarly, cross-modal interactions have been reported for the auditory thalamus (Komura et al., 2005). Because some sensorimotor loops are formed below the cortical level (Diamond et al., 2008), early thalamic cross-modal interactions might speed up the reaction time.

The second mechanism of cross-modal processing involves the activation of neuronal networks in the primary sensory cortices. Visual stimuli reset the phase of network oscillations in the barrel field. When presented alone, light flashes induced a prominent and sustained phase concentration of low-frequency oscillations in all layers of contralateral S1, whereas the effect on the ipsilateral side was much weaker and shorter. Similarly, tactile stimulation caused phase reset in V1. As shown by their augmented power, superimposed fast oscillations $(\beta-\gamma$ frequency band) accumulated at a specific phase of ongoing low-frequency oscillatory activity. Consequently, a co-occurring tactile stimulus arrives during the same phase of ongoing oscillations in S1. The coincidence of a stimulus with a specific (high- or lowexcitability) phase of network oscillations is assumed to increase its processing efficiency (Fries et al., 2001). Similar oscillatory phase reset has been previously proposed as an underlying mech- 
anism of cross-modal interactions in the auditory cortex (Lakatos et al., 2005; Kayser et al., 2008). Both supra-additive auditorysomatosensory and subadditive auditory-visual interactions were associated with a phase resetting of oscillatory activity in the A1 (Lakatos et al., 2007; Thorne et al., 2011). The mechanism of phase reset also shows an incongruency effect because the weaker and shorter visual phase concentration results in a weaker power modulation of oscillatory activity. Because we observed a similar cross-modal phase modulation in S1 and V1, we suggest that phase reset of ongoing oscillations might be a general mechanism of multisensory interactions used by all sensory systems.

Moreover, we elucidated the possible anatomical pathways that mediate the observed phase reset. As shown by retrograde tracing and lidocaine-induced silencing of $\mathrm{V} 1$ activity, direct projections from the V1 to S1 might represent the anatomical substrate of cross-modal modulation of network oscillations. These projections mainly target supragranular layers of the S1 (Paperna and Malach, 1991); and correspondingly, lidocaine had at this depth the strongest effect on the oscillatory phase concentration. Corticocortical projections terminating in layer II/III may boost neurotransmitter release in these layers. Similarly, GABAergic transmission in the supragranular layer has been identified as a prerequisite for phase concentration of ongoing activity and a key cellular mechanism that underlies auditory inhibition of V1 (Iurilli et al., 2012). Taking into account the rather low density of direct projections between $\mathrm{V} 1$ and $\mathrm{S} 1$ and the persistence, even at a lower magnitude, of visually induced phase reset in $\mathrm{S} 1$ granular and infragranular layers after lidocaine silencing of V1, it is very likely that additional mechanisms are meant to amplify and/or complement the cross-modal interplay. The high density of retrogradely labeled neurons in V2 (Fig. $7 B$ ) suggests that higherorder visual cortices that receive feedforward inputs from V1 may be equally involved. However, the cross-modal effects are relayed from V1 to S1 via few stations because the visually induced phase reset in S1 is only slightly delayed compared with the visually evoked response in V1 (42-68 ms vs 37-40 ms). On the other hand, projections from multisensory thalamic nuclei as well as feedback projections from higher-order convergence areas (Theyel et al., 2010) might account for the delayed lidocaineinsensitive peaks of phase concentration.

In contrast, the weaker power modulation and phase reset of S1 activity, which were induced by spatially incongruent stimulation, seem to rely on different mechanisms. In the absence of direct interhemispheric connections between V1 and S1, either ipsilateral projections from the retina to the dorsal LGN (Discenza and Reinagel, 2012) or transcallosal information transfer within V1 or S1 (Genc et al., 2011; Ragert et al., 2011) may account for the phase reset observed when visual and tactile stimuli were presented in opposite hemispheres.

The present results suggest that the visual modulation of S1 activity facilitates the processing of tactile stimuli. Even though the rat state (sleep-like conditions under urethane anesthesia) and the simple stimulation patterns used do not perfectly match the natural multisensory stimulation in behaving animal, the investigation under these controlled conditions identified general principles and mechanisms of cross-modal interactions. As recently shown (Rowland et al., 2012), interactions identified under anesthetized conditions might equally control cross-modal processing in a complex natural environment. We propose that, similar to recent findings on audiovisual detection (Gleiss and Kayser, 2012), access to visual and tactile information is beneficial for rats, supporting perceptual discrimination. Performance in whisker-based discrimination tasks has been reported to critically depend on V1 neuronal firing, even in the dark (Vasconcelos et al., 2011). Similarly, looking at the arm at which a tactile stimulus was applied reduced the two-point discrimination in humans (Kennett et al., 2001). This visual enhancement of touch was found to involve visual modulation of S1 (Serino et al., 2009). We propose that both cross-modal interactions at subcortical (thalamic) level and the oscillatory entrainment of primary sensory cortices via direct connectivity are powerful instruments of information processing, which help individuals to detect and localize the most salient and possibly relevant events in the environment.

\section{References}

Arnal LH, Wyart V, Giraud AL (2011) Transitions in neural oscillations reflect prediction errors generated in audiovisual speech. Nat Neurosci 14:797-801. CrossRef Medline

Barth DS, Goldberg N, Brett B, Di S (1995) The spatiotemporal organization of auditory, visual, and auditory-visual evoked potentials in rat cortex. Brain Res 678:177-190. CrossRef Medline

Beer AL, Plank T, Greenlee MW (2011) Diffusion tensor imaging shows white matter tracts between human auditory and visual cortex. Exp Brain Res 213:299-308. CrossRef Medline

Botvinick M, Cohen J (1998) Rubber hands "feel" touch that eyes see. Nature 391:756. CrossRef Medline

Brockmann MD, Pöschel B, Cichon N, Hanganu-Opatz IL (2011) Coupled oscillations mediate directed interactions between prefrontal cortex and hippocampus of the neonatal rat. Neuron 71:332-347. CrossRef Medline

Cappe C, Barone P (2005) Heteromodal connections supporting multisensory integration at low levels of cortical processing in the monkey. Eur J Neurosci 22:2886-2902. CrossRef Medline

Cappe C, Rouiller EM, Barone P (2009) Multisensory anatomical pathways. Hear Res 258:28-36. CrossRef Medline

Chapin JK, Lin CS (1990) The somatic sensory cortex of the rat. In: The cerebral cortex of the rat (Kolb B, Tees RC, eds), pp 341-380. Cambridge: MIT.

Civillico EF, Contreras D (2012) Spatiotemporal properties of sensory responses in vivo are strongly dependent on network context. Front Syst Neurosci 6:25. CrossRef Medline

Clavagnier S, Falchier A, Kennedy H (2004) Long-distance feedback projections to area $\mathrm{V} 1$ : implications for multisensory integration, spatial awareness, and visual consciousness. Cogn Affect Behav Neurosci 4:117-126. CrossRef Medline

Clement EA, Richard A, Thwaites M, Ailon J, Peters S, Dickson CT (2008) Cyclic and sleep-like spontaneous alternations of brain state under urethane anaesthesia. PLoS ONE 3:e2004. CrossRef Medline

Cohen L, Rothschild G, Mizrahi A (2011) Multisensory integration of natural odors and sounds in the auditory cortex. Neuron 72:357-369. CrossRef Medline

Deeg KE, Aizenman CD (2011) Sensory modality-specific homeostatic plasticity in the developing optic tectum. Nat Neurosci 14:548-550. CrossRef Medline

Diamond ME, von Heimendahl M, Knutsen PM, Kleinfeld D, Ahissar E (2008) "Where" and "what" in the whisker sensorimotor system. Nat Rev Neurosci 9:601-612. CrossRef Medline

Discenza CB, Reinagel P (2012) Dorsal lateral geniculate substructure in the Long-Evans rat: a cholera toxin B subunit study. Front Neuroanat 6:40. CrossRef Medline

Driver J, Spence C (2000) Multisensory perception: beyond modularity and convergence. Curr Biol 10:R731-R735. CrossRef Medline

Fries P, Neuenschwander S, Engel AK, Goebel R, Singer W (2001) Rapid feature selective neuronal synchronization through correlated latency shifting. Nat Neurosci 4:194-200. CrossRef Medline

Frostig RD, Xiong Y, Chen-Bee CH, Kvasnák E, Stehberg J (2008) Largescale organization of rat sensorimotor cortex based on a motif of large activation spreads. J Neurosci 28:13274-13284. CrossRef Medline

Fu KM, Johnston TA, Shah AS, Arnold L, Smiley J, Hackett TA, Garraghty PE, Schroeder CE (2003) Auditory cortical neurons respond to somatosensory stimulation. J Neurosci 23:7510-7515. Medline

Genç E, Bergmann J, Tong F, Blake R, Singer W, Kohler A (2011) Callosal connections of primary visual cortex predict the spatial spreading of binocular rivalry across the visual hemifields. Front Hum Neurosci 5:161. CrossRef Medline 
Ghazanfar AA, Schroeder CE (2006) Is neocortex essentially multisensory? Trends Cogn Sci 10:278-285. CrossRef Medline

Gleiss S, Kayser C (2012) Audio-visual detection benefits in the rat. PLoS ONE 7:e45677. CrossRef Medline

Guzman-Martinez E, Ortega L, Grabowecky M, Mossbridge J, Suzuki S (2012) Interactive coding of visual spatial frequency and auditory amplitude-modulation rate. Curr Biol 22:383-388. CrossRef Medline

Herkenham M (1980) Laminar organization of thalamic projections to the rat neocortex. Science 207:532-535. CrossRef Medline

Iurilli G, Ghezzi D, Olcese U, Lassi G, Nazzaro C, Tonini R, Tucci V, Benfenati F, Medini P (2012) Sound-driven synaptic inhibition in primary visual cortex. Neuron 73:814-828. CrossRef Medline

Janiesch PC, Krüger HS, Pöschel B, Hanganu-Opatz IL (2011) Cholinergic control in developing prefrontal-hippocampal networks. J Neurosci 31: 17955-17970. CrossRef Medline

Kayser C, Petkov CI, Logothetis NK (2007) Tuning to sound frequency in auditory field potentials. J Neurophysiol 98:1806-1809. CrossRef Medline

Kayser C, Petkov CI, Logothetis NK (2008) Visual modulation of neurons in auditory cortex. Cereb Cortex 18:1560-1574. CrossRef Medline

Kennett S, Taylor-Clarke M, Haggard P (2001) Noninformative vision improves the spatial resolution of touch in humans. Curr Biol 11:1188-1191. CrossRef Medline

Komura Y, Tamura R, Uwano T, Nishijo H, Ono T (2005) Auditory thalamus integrates visual inputs into behavioral gains. Nat Neurosci 8:1203-1209. CrossRef Medline

Lakatos P, Shah AS, Knuth KH, Ulbert I, Karmos G, Schroeder CE (2005) An oscillatory hierarchy controlling neuronal excitability and stimulus processing in the auditory cortex. J Neurophysiol 94:1904-1911. CrossRef Medline

Lakatos P, Chen CM, O'Connell MN, Mills A, Schroeder CE (2007) Neuronal oscillations and multisensory interaction in primary auditory cortex. Neuron 53:279-292. CrossRef Medline

Meredith MA (2002) On the neuronal basis for multisensory convergence: a brief overview. Brain Res Cogn Brain Res 14:31-40. CrossRef Medline

Meredith MA, Stein BE (1983) Interactions among converging sensory inputs in the superior colliculus. Science 221:389-391. CrossRef Medline

Miller BT, D'Esposito M (2005) Searching for "the top" in top-down control. Neuron 48:535-538. CrossRef Medline

Nicholson C, Freeman JA (1975) Theory of current source-density analysis and determination of conductivity tensor for anuran cerebellum. J Neurophysiol 38:356-368. Medline

Noesselt T, Tyll S, Boehler CN, Budinger E, Heinze HJ, Driver J (2010) Sound-induced enhancement of low-intensity vision: multisensory influences on human sensory-specific cortices and thalamic bodies relate to perceptual enhancement of visual detection sensitivity. J Neurosci 30: 13609-13623. CrossRef Medline

Paperna T, Malach R (1991) Patterns of sensory intermodality relationships in the cerebral cortex of the rat. J Comp Neurol 308:432-456. CrossRef Medline

Prusky GT, Harker KT, Douglas RM, Whishaw IQ (2002) Variation in visual acuity within pigmented, and between pigmented and albino rat strains. Behav Brain Res 136:339-348. CrossRef Medline

Ragert P, Nierhaus T, Cohen LG, Villringer A (2011) Interhemispheric interactions between the human primary somatosensory cortices. PLoS One 6:e16150. CrossRef Medline
Rodgers KM, Benison AM, Klein A, Barth DS (2008) Auditory, somatosensory, and multisensory insular cortex in the rat. Cereb Cortex 18:2941-2951. CrossRef Medline

Rowland BA, Perrault TJ, Keniston LP, Vaughan JW, Stein BE (2012) Temporal profiles of multisensory integration in the awake cat superior colliculus. SFN Meeting 2012 New Orleans, Louisiana.

Roy NC, Bessaih T, Contreras D (2011) Comprehensive mapping of whisker-evoked responses reveals broad, sharply tuned thalamocortical input to layer 4 of barrel cortex. J Neurophysiol 105:2421-2437. CrossRef Medline

Schmued LC, Fallon JH (1986) Fluoro-Gold: a new fluorescent retrograde axonal tracer with numerous unique properties. Brain Res 377:147-154. CrossRef Medline

Schroeder CE, Mehta AD, Givre SJ (1998) A spatiotemporal profile of visual system activation revealed by current source density analysis in the awake macaque. Cereb Cortex 8:575-592. CrossRef Medline

Senkowski D, Schneider TR, Foxe JJ, Engel AK (2008) Crossmodal binding through neural coherence: implications for multisensory processing. Trends Neurosci 31:401-409. CrossRef Medline

Serino A, Padiglioni S, Haggard P, Làdavas E (2009) Seeing the hand boosts feeling on the cheek. Cortex 45:602-609. CrossRef Medline

Stein BE (2012) The new handbook of multisensory processing. Cambridge, MA: MIT.

Stein BE, Rowland BA (2011) Organization and plasticity in multisensory integration: early and late experience affects its governing principles. Prog Brain Res 191:145-163. CrossRef Medline

Stein BE, Stanford TR (2008) Multisensory integration: current issues from the perspective of the single neuron. Nat Rev Neurosci 9:255-266. CrossRef Medline

Sugitani M (1979) Electrophysiological and sensory properties of the thalamic reticular neurones related to somatic sensation in rats. J Physiol 290:79-95. Medline

Tehovnik EJ, Sommer MA (1997) Effective spread and timecourse of neural inactivation caused by lidocaine injection in monkey cerebral cortex. J Neurosci Methods 74:17-26. CrossRef Medline

Theyel BB, Llano DA, Sherman SM (2010) The corticothalamocortical circuit drives higher-order cortex in the mouse. Nat Neurosci 13:84-88. CrossRef Medline

Thorne JD, De Vos M, Viola FC, Debener S (2011) Cross-modal phase reset predicts auditory task performance in humans. J Neurosci 31:3853-3861. CrossRef Medline

Tyll S, Budinger E, Noesselt T (2011) Thalamic influences on multisensory integration. Commun Integr Biol 4:378-381. CrossRef Medline

Vasconcelos N, Pantoja J, Belchior H, Caixeta FV, Faber J, Freire MA, Cota VR, Anibal de Macedo E, Laplagne DA, Gomes HM, Ribeiro S (2011) Cross-modal responses in the primary visual cortex encode complex objects and correlate with tactile discrimination. Proc Natl Acad Sci U S A 108:15408-15413. CrossRef Medline

Wang XJ (2010) Neurophysiological and computational principles of cortical rhythms in cognition. Physiol Rev 90:1195-1268. CrossRef Medline

Wepsic JG (1966) Multimodal sensory activation of cells in the magnocellular medial geniculate nucleus. Exp Neurol 15:299-318. CrossRef Medline

Wise SP, Jones EG (1978) Developmental studies of thalamocortical and commissural connections in the rat somatic sensory cortex. J Comp Neurol 178:187-208. CrossRef Medline 